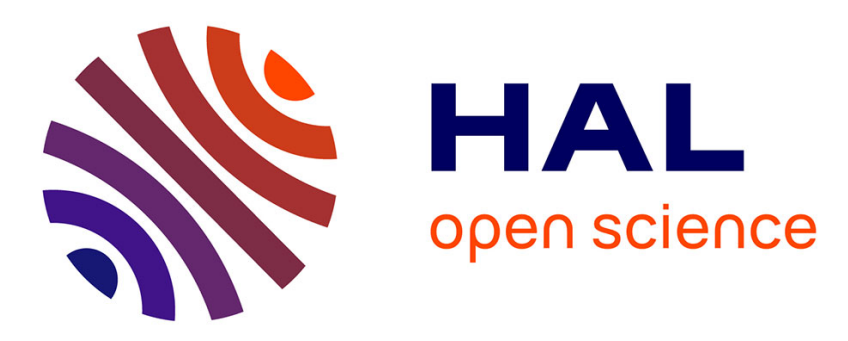

\title{
Iterative methods for the canonical decomposition of multi-way arrays: Application to blind underdetermined mixture identification
}

\author{
Ahmad Karfoul, Laurent Albera, Lieven de Lathauwer
}

\section{To cite this version:}

Ahmad Karfoul, Laurent Albera, Lieven de Lathauwer. Iterative methods for the canonical decomposition of multi-way arrays: Application to blind underdetermined mixture identification. Signal Processing, 2011, 91 (8), pp.1789-1802. 10.1016/j.sigpro.2011.02.003 . hal-00591824

\section{HAL Id: hal-00591824 \\ https://hal.science/hal-00591824}

Submitted on 13 May 2011

HAL is a multi-disciplinary open access archive for the deposit and dissemination of scientific research documents, whether they are published or not. The documents may come from teaching and research institutions in France or abroad, or from public or private research centers.
L'archive ouverte pluridisciplinaire HAL, est destinée au dépôt et à la diffusion de documents scientifiques de niveau recherche, publiés ou non, émanant des établissements d'enseignement et de recherche français ou étrangers, des laboratoires publics ou privés. 


\title{
Iterative methods for the canonical decomposition of multi-way arrays: application to blind underdetermined mixture identification
}

\author{
Ahmad Karfoul $^{\mathrm{a}}$, Laurent Albera ${ }^{\mathrm{b}, \mathrm{c}}$, Lieven De Lathauwer ${ }^{\mathrm{d}, \mathrm{e}}$ \\ ${ }^{a}$ AL-Baath University, Faculty of Mechanical and Electrical Engineering, PB. 2244, Homs, Syria \\ ${ }^{b}$ Inserm, UMR 642, Rennes, F-35000, France \\ ${ }^{c}$ LTSI Laboratory, University of Rennes 1, Rennes, F-35000, France \\ ${ }^{d}$ K. U. Leuven Campus Kortrijk, Group Science, Engineering and Technology, Kortrijk, Belgium \\ ${ }^{e}$ K. U. Leuven, E. E. Dept. (ESAT), Leuven, Belgium
}

\begin{abstract}
Two main drawbacks can be stated in the Alternating Least Square (ALS) algorithm used to fit the CANonical Decomposition (CAND) of multi-way arrays. First its slow convergence caused by the presence of collinearity between factors in the multi-way array it decomposes. Second its blindness to Hermitian symmetries of the considered arrays. Enhanced Line Search (ELS) scheme was found to be a good way to cope with the slow convergence of the ALS algorithm together with a partial use of the Hermitian symmetry. However, to our knowledge, required equations to perform the latter scheme are only given in the case of third and fifth order arrays. Therefore, our first contribution consists in generalizing the ELS procedure to the case of complex arrays of any order greater than three. Our second contribution is another improvement of the ALS scheme, able to profit from Hermitianity and positive semi-definiteness of the considered arrays. It consists in resorting to the CAND of a third order array having one unitary loading matrix. An iterative algorithm is then proposed alternating between Procrustes problem solving and the computation of rank-one matrix approximations.
\end{abstract}

Keywords: CANonical Decomposition (CAND), PARAllel FACtor analysis (PARAFAC), multi-way data analysis, Alternating Least Squares (ALS), Hermitian multi-way arrays, Blind Underdetermined Mixture Identification (BUMI)

\footnotetext{
Email addresses: AKarfoul@gmail.com (Ahmad Karfoul), laurent.albera@univ-rennes1.fr (Laurent Albera), Lieven.DeLathauwer@kuleuven-kortrijk.be (Lieven De Lathauwer)

Preprint submitted to Signal Processing 


\section{Introduction}

CANonical Decomposition (CAND) of a Higher Order (HO) array is the decomposition of the array as a linear combination of a minimal number of rank-1 terms. Recall that HO arrays are those arrays whose elements are addressed by more than two indices. In addition to the Tucker decomposition/HO Singular Value Decomposition (HOSVD)[1, 2], CAND is another possible generalization of SVD [3] to HO arrays but without any orthogonal constraint. According to its definition, CAND relies essentially on the concept of HO array rank and low-rank decomposition, and its uniqueness properties mark its specific characteristic. The symmetric variant of the CAND defined when those rank-1 terms are symmetric is going back to the nineteenth century in the context of invariant theory [4]. Indeed, each symmetric $2 q$-th $(q \geqslant 1)$ order array of dimension $N$ is associated to a $2 q$-th order homogeneous polynomial with $N$ variables. Then the CAND of the considered HO arrays is rephrased in terms of polynomials as the sum of $N d$-th powers of linear forms. In fact, the unsymmetric variant was first tackled by Hitchcock in 1927 $[5,6]$. Since then, the use of the CAND concept grows together with the technological innovations that make possible the acquisition of multi-dimensional measurements with meaningful form such as in psychometrics [7] where the term CAND is employed, in chemometrics [8] where the term PARAFAC (PARallel FACtor analysis) is used instead, in exploratory data analysis [9] and in signal processing area [10]. Even when the observation diversity is not sufficient, meaningful HO arrays can still be formed by resorting to HO Statistics (HOS) of the data such as in radiocommunications $[11,12,13,14]$.

A multitude of methods have then been developed to fit the CAND of a given HO array. Some of them are semi-algebraic and others are iterative. For example, author in [15] shows that CAND of third and fourth order arrays can be performed by means of a joint diagonalization of a set of matrices leading to a new uniqueness condition less restrictive than the one defined by Kruskal [16] (see section 2). In addition, we can find the Direct TriLinear Decomposition algorithm (DTLD) and the Generalized Rank Annihilation Method (GRAM) [17]. Both the DTLD and the GRAM approaches are based on a generalized eigenvalue problem. Authors in [18] show that CAND can be solved by means of a simultaneous generalized Schur decomposition. On the other hand, authors in [13] exploit redundancies in the considered Hermitian positive semi- 
definite arrays to perform CAND. Regarding the iterative approaches, several methods were proposed such as the non-linear conjugate gradient approach [19], the LevenbergMarquardt method [20, 21, 22, 23], the Alternating Least Squares (ALS) algorithms [24], the Alternating Slice-wise Diagonalization (ASD) [25] and Self Weighted Alternating TriLinear Decomposition (SWATLD) [26], to cite a few. Due to its simplicity the ALS algorithm is commonly used to fit the CAND of a given HO array. However, these iterative algorithms do not benefit from the symmetries of the considered arrays, which occur for instance when we resort to HOS [11, 12, 13, 14]. Moreover, several studies were reported showing how the ALS convergence is slowed down in the presence of certain symmetries [23, 27]. Bro and Harshman [28, 29] were the first to state that when ALS is slow, there exists cycles of convergence defined by a unique direction. In each cycle, factors evolve in the same direction to the final solution (see [27] and the references therein). Enhanced Line Search (ELS) scheme was found to be a good tool to cope with the problem of slow convergence $[27,30]$. Indeed, ELS relies on an optimal extrapolation of factors many iterations ahead (see section 3.1). This extrapolation allows the algorithm to attain the final solution of a given cycle in a few iterations. To our knowledge, the required equations to perform the ELS step were only given in the case of third order $[30,27]$ and fifth order [31] arrays in spite of the need for general equations allowing us to decompose for instance fourth [14] and sixth [13] order arrays.

The goal of this paper is twofold. First and following the same technique proposed in [30], a generalization of the ELSALS approach to complex arrays of any HO is presented (the real case is then straightforward). Second, a new ALS-like method, named CANDHAP, is proposed to canonically decompose Hermitian positive semi-definite Arrays based on Procrustes problem. For such arrays, CANDHAP allows for a reduction in the dimensionality of the problem. Indeed, it reduces the CAND of a $2 q$-th $(q \geqslant 2)$ order Hermitian positive semi-definite array to the CAND of a third order array having one unitary loading matrix. The latter CAND is then originally performed by alternating between Procrustes problem solving [32] and the computation of rank-one matrix approximations. Consequently a higher accuracy, a faster convergence and a lower numerical complexity than the ELSALS algorithm are guaranteed, as shown in our numerical experiments, provided that the considered array is Hermitian positive semi-definite. Preliminary ver- 
sions of CANDHAP appeared in conference papers [33, 34]. However, this journal paper presents a full and detailed study of the proposed algorithm in addition to the generalization of the ELSALS method to any order equal or higher than three. Application of the proposed techniques to the Blind Underdetermined Mixture Identification (BUMI) problem $[13,35,36]$ is also considered.

\section{Multilinear algebra preliminaries}

In this paper, vectors, matrices and $\mathrm{HO}$ arrays are denoted with bold lowercase letters $(\boldsymbol{a}, \boldsymbol{b}, \cdots)$, with bold uppercase letters $(\boldsymbol{A}, \boldsymbol{B}, \cdots)$ and with calligraphic letters $(\mathcal{A}, \mathcal{B}, \cdots)$, respectively. In addition, lower-order parts of a given structure are considered as scalars and follow as a result the same notation of their structures. For instance, the entry with row $i$ and column $j$ in a matrix $\boldsymbol{A}$ is symbolized by $A_{i, j}$ and the $\left(i_{1}, i_{2}, \cdots, i_{q}\right)$-th component of a $q$-th order array $\mathcal{B}$ is symbolized by $\mathcal{B}_{i_{1}, i_{2}, \cdots, i_{q}}$. Sometimes we will use the MATLAB colon/row notation to indicate sub-matrices of a given matrix or sub-arrays of a given $\mathrm{HO}$ array. Upper bounds (e.g., $n=1,2, \cdots, N$ ) will be denoted by italic capital letters. Recall that the $p$-th column vector of the Khatri-Rao product $\boldsymbol{A} \oslash \boldsymbol{B}$ is given by $\left[A_{1, p} \boldsymbol{b}_{p}^{\top}, \cdots, A_{P, p} \boldsymbol{b}_{p}^{\top}\right]^{\top}$ where $A_{n, p}$ and $\boldsymbol{b}_{p}$ denote the $(n, p)$-th component of $\boldsymbol{A}$ and the $p$-th column vector of $\boldsymbol{B}$, respectively. Thus $\boldsymbol{A}^{\oslash q}$ will denote the product $\boldsymbol{A} \oslash \cdots \oslash \boldsymbol{A}$ where $\oslash$ occurs $q-1$ times. Furthermore, the subscripts $*,{ }^{\dagger},{ }^{\top},{ }^{H}$ and $\|\cdot\|_{F}$ stand for the complex conjugate, the Moore-Penrose pseudoinverse, the transpose, the complex conjugated transpose and the Frobenius norm operators, respectively. In addition, the $(N \times N)$ identity matrix is denoted by $\mathbf{I}_{N}$ while $|a|$ is the absolute value of $a$. Finally, the Kronecker operator $\delta_{a, b}$ is defined such as $\delta_{a, b}=1$ if $a=b$ and 0 otherwise. Now, let us consider some basic definitions in multilinear algebra that will be used throughout the paper $[15,37]$.

Definition 1. A rank-1 $q$-th order array $\mathcal{T} \in \mathbb{C}^{N_{1} \times \cdots \times N_{q}}$ is equal to the outer product $\boldsymbol{a}^{(1)} \circ \cdots \circ \boldsymbol{a}^{(q)}$ of $q$ vectors $\boldsymbol{a}^{(i)} \in \mathbb{C}^{N_{i}}$ where each component of $\mathcal{T}$ is defined by $\mathcal{T}_{i_{1}, \cdots, i_{q}}=$ $a_{i_{1}}^{(1)} \cdots a_{i_{q}}^{(q)}$. Then $\boldsymbol{a}^{\circ q}$ is the outer product of $q$ vectors $\boldsymbol{a}$.

Definition 2. The $i$-mode product of a $q$-th order array $\mathcal{T} \in \mathbb{C}^{N_{1} \times \cdots \times N_{q}}$ and a matrix 
$\boldsymbol{U} \in \mathbb{C}^{M \times N_{i}}$ is a q-th order array of $\mathbb{C}^{N_{1} \times \cdots \times N_{i-1} \times M \times N_{i+1} \times \cdots \times N_{q}}$ with components given by:

$$
\left(\mathcal{T} \times_{i} \boldsymbol{U}\right)_{n_{1}, \cdots, n_{i-1}, m, n_{i+1}, \cdots, n_{2 q}}=\sum_{n_{i}=1}^{N_{i}} \mathcal{T}_{n_{1}, \cdots, n_{i}, \cdots, n_{2 q}} U_{m, n_{i}}
$$

The rank of a HO array always exists and is defined by:

Definition 3. The rank of a q-th order $(q \geqslant 2)$ array $\mathcal{T} \in \mathbb{C}^{N_{1} \times \cdots \times N_{q}}$, denoted by $\operatorname{rk}(\mathcal{T})$, is the minimal number of rank-1 q-th order arrays that yield $\mathcal{T}$ in a linear combination.

It is noteworthy that despite the similarity between matrices and HO arrays, the rank of multi-way arrays can exceed its smallest dimension. For instance, the rank of a third order array of size $(3 \times 3 \times 3)$ is generically equal to 5 [38]. Note that a property is called generic when it holds everywhere except for a set of Lebesgue measure zero. From definitions 1 and 3, CAND can be defined as follows [27, 30, 39]:

Definition 4. The CAND of a complex $q$-th order $(q \geqslant 2)$ array $\mathcal{T}$ is the linear combination of $P=\operatorname{rk}(\mathcal{T})$ rank-1 $q$-th order arrays that yields $\mathcal{T}$ exactly:

$$
\mathcal{T}=\sum_{p=1}^{P} \lambda_{p} \boldsymbol{a}_{p}^{(1)} \circ \boldsymbol{a}_{p}^{(2)} \circ \cdots \circ \boldsymbol{a}_{p}^{(q)}
$$

with $\lambda_{p} \in \mathbb{C}$ and where the $q$ matrices $\boldsymbol{A}^{(i)}=\left[\boldsymbol{a}_{1}^{(i)}, \cdots, \boldsymbol{a}_{P}^{(i)}\right]$ are called the loading matrices of $\mathcal{T}$. Sometimes we use the notation $\mathcal{T}\left(\boldsymbol{A}^{(1)}, \cdots, \boldsymbol{A}^{(q)}\right)$ to refer to the HO array $\mathcal{T}$ with its loading matrices $\boldsymbol{A}^{(1)}, \cdots, \boldsymbol{A}^{(q)}$.

Definition 4 shows that the different rank-1 terms can be permuted and scaled/counterscaled within the rank-1 term without modifying the sum. A CAND is then considered to be essentially unique when it is only subject to these trivial indeterminacies. Several studies were performed in order to find the appropriate conditions for CAND's uniqueness. The first one was tackled by Cattell in 1944 [40] and addressed later by Harshman in 1970 [29]. Moreover, an important result on the CAND uniqueness was obtained by Kruskal in 1977 [16] which is generalized to arrays with arbitrary order $q,(q \geqslant 3)$, by Sidiropoulos and Bro [41]. Indeed, this condition shows that the CAND of a $q$-th order array $\mathcal{T}$ is essentially unique when the following sufficient condition is satisfied [41]:

$$
2 P+q-1 \leqslant \sum_{i=1}^{q} \operatorname{rk}_{\mathrm{k}}\left(\boldsymbol{A}^{(i)}\right)
$$


where $\operatorname{rk}_{\mathrm{k}}(\boldsymbol{B})$ is the Kruskal rank of $\boldsymbol{B}$, which is defined as the largest number $z$ such that every subset of $z$ columns of $\boldsymbol{B}$ is linearly independent. Kruskal's condition (3) was first proposed for real third order arrays. Later, it was extended to arrays with order higher than three [42] and for complex HO arrays [43]. Alternative less restrictive conditions were also proposed dealing with special third order arrays [44, 15]. Indeed authors in $[44,15]$ addressed the case of third order arrays with one loading matrix being full column rank. On the other hand, authors in [45] stated that the CAND of a symmetric HO array is essentially unique with probability one when the dimensions of the latter does not exceed its order. Generically, it can be stated that each HO array has a unique CAND when its rank falls between the rank defined by Kruskal's condition (3) and its generic rank (or its smallest typical rank in the case of real tensors) [23]. The definition of the CAND above (2) includes the case of Hermitian HO arrays defined hereafter.

Definition 5. A P-rank q-way $(q \geqslant 2)$ array $\mathcal{T} \in \mathbb{C}^{N_{1} \times \cdots \times N_{q}}$ will be called Hermitian if i) its order $q$ is even, ii) it is cubic [46], say all its dimensions are equal, iii) it admits a CAND with as many conjugate terms of the same factor as non-conjugate ones and iv) the weights $\lambda_{p}$ 's of its CAND have all values in the real field:

$$
\mathcal{T}=\sum_{p=1}^{P} \lambda_{p} \boldsymbol{a}_{p}^{\circ q / 2} \circ \boldsymbol{a}_{p}^{* \circ q / 2}
$$

Particular Hermitian arrays are often used in signal processing known under the name of cumulants $[13,35,36,47]$. They allow for a blind identification of static mixture of independent electromagnetic waves, say to solve the BUMI problem. In some practical contexts such as radiocommunications, human-made waves have generally positive marginal Sixth Order ( $\mathrm{SixO}$ ) cumulants, implying the positive semi-definiteness of the SixO cumulant arrays of every static mixture of such sources.

Definition 6. A HO Hermitian array is called positive semi-definite if the weights $\lambda_{p}$ 's of its CAND (4) are positive.

Definition 7. Let $\mathcal{T}$ be a $2 q$-th order $(q \geqslant 2)$ array whose dimensions are all equal to $N$. Let also $\lfloor q / 2\rfloor$ and $\lceil q / 2\rceil$ be the lower and the upper integer parts of $q / 2$, respectively. Then the $(i, j)$-th component of the unfolding matrix $\operatorname{mat}_{1}(\mathcal{T})$ of size $\left(N^{q} \times N^{q}\right)$ is 
given by $\left(\operatorname{mat}_{1}(\mathcal{T})\right)_{i, j}=\mathcal{T}_{n_{1}, \cdots, n_{[q / 2]}, n_{[q / 2]+1}, \cdots, n_{q}, n_{q+1}, \cdots, n_{q+[q / 2]}, n_{q+[q / 2]+1}, \cdots, n_{2 q}}$ where $i=$ $\left(n_{1}-1\right) N^{q-1}+\cdots+\left(n_{\lceil q / 2\rceil}-1\right) N^{\lfloor q / 2\rfloor}+\left(n_{q+\lceil q / 2\rceil+1}-1\right) N^{\lfloor q / 2\rfloor-1}+\cdots+\left(n_{2 q-1}-1\right) N+n_{2 q}$ and $j=\left(n_{q+1}-1\right) N^{q-1}+\cdots+\left(n_{q+\lceil q / 2\rceil}-1\right) N^{\lfloor q / 2\rfloor}+\left(n_{\lceil q / 2\rceil+1}-1\right) N^{\lfloor q / 2\rfloor-1}+\cdots+\left(n_{q-1}-1\right) N+n_{q}$. Then the unmat ${ }_{1}$ operator is defined such as unmat $_{1}\left(\operatorname{mat}_{1}(\mathcal{T})\right)=\mathcal{T}$.

Example 1. Let $\mathcal{T}$ be a square 6 -th order array of dimensions $N$. Then the $(i, j)$-th component of its square unfolding matrix $\operatorname{mat}_{1}(\mathcal{T})$ is given by $\left(\operatorname{mat}_{1}(\mathcal{T})\right)_{i, j}=\mathcal{T}_{n_{1}, n_{2}, n_{3}, n_{4}, n_{5}, n_{6}}$ where $i=\left(n_{1}-1\right) N^{2}+\left(n_{2}-1\right) N+n_{6}$ and $j=\left(n_{4}-1\right) N^{2}+\left(n_{5}-1\right) N+n_{3}$.

Another way of unfolding HO arrays is presented hereafter.

Definition 8. Let $\mathcal{T} \in \mathbb{C}^{N_{1} \times \cdots \times N_{q}}$ be a q-th order array. Then the $\left(n_{i}, m\right)$-th component of the unfolding matrix mat ${ }_{2}^{(i)}(\mathcal{T}) \in \mathbb{C}^{N_{i} \times N_{i+1} \cdots N_{q} N_{1} \cdots N_{i-1}}$ associated to the $i$-th mode $(1 \leqslant i \leqslant q)$ of $\mathcal{T}$ is given by $\left(\operatorname{mat}_{2}^{(i)}(\mathcal{T})\right)_{n_{i}, m}=\mathcal{T}_{n_{1}, \cdots, n_{i-1}, n_{i}, n_{i+1}, \cdots, n_{q}}$ where $m=\left(n_{i+1}-\right.$ 1) $N_{i+2} \cdots N_{q} N_{1} \cdots N_{i-1}+\left(n_{i+2}-1\right) N_{i+3} \cdots N_{q} N_{1} \cdots N_{i-1}+\cdots+\left(n_{q}-1\right) N_{1} N_{2} \cdots N_{i-1}+$ $\left(n_{1}-1\right) N_{2} N_{3} \cdots N_{i-1}+\left(n_{2}-1\right) N_{3} N_{4} \cdots N_{i-1}+\cdots+n_{i-1}$. Then the unmat ${ }_{2}^{(i)}$ operator is defined such as unmat ${ }_{2}^{(i)}\left(\operatorname{mat}_{2}^{(i)}(\mathcal{T})\right)=\mathcal{T}$.

Example 2. Let $\mathcal{T} \in \mathbb{C}^{N_{1} \times N_{2} \times N_{3}}$ be a third order array whose loading matrices are $\boldsymbol{A}^{(1)}, \boldsymbol{A}^{(2)}$ and $\boldsymbol{A}^{(3)}$. Then we have $\operatorname{mat}_{2}^{(1)}(\mathcal{T})=\boldsymbol{A}^{(1)}\left(\boldsymbol{A}^{(2)} \oslash \boldsymbol{A}^{(3)}\right)^{\top}$, $\operatorname{mat}_{2}^{(2)}(\mathcal{T})=$ $\boldsymbol{A}^{(2)}\left(\boldsymbol{A}^{(3)} \oslash \boldsymbol{A}^{(1)}\right)^{\top}$ and $\operatorname{mat}_{2}^{(3)}(\mathcal{T})=\boldsymbol{A}^{(3)}\left(\boldsymbol{A}^{(1)} \oslash \boldsymbol{A}^{(2)}\right)^{\top}$.

Definition 9. Let $\mathcal{T} \in \mathbb{C}^{N_{1} \times \cdots \times N_{q}}$ be a q-th order $(q \geqslant 2)$ array. Then the $m$-th element of its vector representation, denoted by $\operatorname{vec}(\mathcal{T}) \in \mathbb{C}^{N_{1} N_{2} \cdots N_{q}}$, is given by $(\operatorname{vec}(\mathcal{T}))_{m}=$ $\mathcal{T}_{n_{1}, \cdots, n_{q}}$ where $m=\left(n_{1}-1\right) N_{2} \cdots N_{q}+\left(n_{2}-1\right) N_{3} \cdots N_{q}+\cdots+n_{q}$, for $1 \leqslant n_{i} \leqslant N_{i}$ with $i \in\{1, \cdots, q\}$. Conversely the unvec operator is defined such as unvec $(\operatorname{vec}(\mathcal{T}))=\mathcal{T}$.

\section{ALS-like techniques for the CAND of multi-way arrays}

Due to its simplicity, the ALS algorithm is commonly used to fit the CAND of a given $q$-th order array $\mathcal{T}\left(\boldsymbol{A}^{(1)}, \cdots, \boldsymbol{A}^{(q)}\right)$. At each iteration of this algorithm, the $q$ loading matrices are estimated one at a time:

$$
\boldsymbol{A}_{i t}^{(i)}=\operatorname{mat}_{2}^{(\mathrm{i})}(\boldsymbol{\mathcal { T }})\left[\left(\boldsymbol{A}_{i t-1}^{(i+1)} \oslash \cdots \oslash \boldsymbol{A}_{i t-1}^{(q)} \oslash \boldsymbol{A}_{i t}^{(1)} \oslash \cdots \oslash \boldsymbol{A}_{i t}^{(i-1)}\right)^{\mathrm{T}}\right]^{\dagger}
$$


where $\boldsymbol{A}_{i t}^{(i)}$ denotes the estimate of $\boldsymbol{A}^{(i)}$ at the $i t$-th iteration. In the presence of collinearity between factors, the convergence speed of the ALS algorithm is slowed down $[23,27,30,28,29]$. As mentioned above, this is due to some convergence cycles wherein the ALS spends a lot of iterations to get the final solution of each cycle. To cope with this slowness, Harshman and Bro [28, 29] have proposed a linear search scheme wherein loading matrices are estimated many iterations ahead. For a given direction defined by a convergence cycle, this estimation process is performed using a predefined step size called relaxation factor. Instead of giving this relaxation factor a fixed value, as done by Harshman and Bro, authors in [27] proposed to compute it optimally giving rise to the Enhanced Line Search (ELS) scheme. However, in the ELS procedure [27], the relaxation factor is still supposed to be real when dealing with complex HO arrays, which may be suboptimal when complex arrays are processed. Therefore, an alternative solution was proposed in [30], which consists of considering the relaxation factor as a complex number when dealing with complex HO arrays. Nevertheless, this approach is limited to third [30] and fifth order arrays [31].

\subsection{A generalized Enhanced Line Search scheme for complex ALS-like algorithms}

We propose in this section to extend the ELS procedure using a relaxation factor to the case of complex arrays of any order greater than three. The generalized ELS procedure to $q$-th $(q \geqslant 1)$ order arrays is defined as following:

Definition 10. The ELS procedure for ALS-like algorithms consists, for a given complex $q$-way array $\mathcal{T}$, in looking for the optimal relaxation factors, $\rho^{(i)}(1 \leqslant i \leqslant q)$ that minimize, the following criterion [27, 30]:

$$
\Psi(\rho)=\left\|\boldsymbol{T}^{(q)}-\boldsymbol{A}_{\text {new }}^{(q)}\left(\boldsymbol{A}_{\text {new }}^{(1)} \oslash \cdots \oslash \boldsymbol{A}_{\text {new }}^{(q-1)}\right)^{\top}\right\|_{F}^{2}
$$

where $\boldsymbol{T}^{(q)}=\operatorname{mat}_{2}^{(q)}(\boldsymbol{T}), \boldsymbol{A}_{\text {new }}^{(i)}=\boldsymbol{A}_{i t-2}^{(i)}+\rho^{(i)} \boldsymbol{G}_{\boldsymbol{A}}^{(i)}(1 \leqslant i \leqslant q)$ is the matrix that will be used at the it-th iteration instead of $\boldsymbol{A}_{\mathrm{it-1}}^{(i)}$ and $\boldsymbol{G}_{\boldsymbol{A}}^{(i)}$ defines the search direction.

For real 3-way arrays, the optimal relaxation factors are obtained by solving a system of three polynomials in three unknowns $\left(\rho^{(1)}, \rho^{(2)}, \rho^{(3)}\right)[27]$. However, this solution is well-known to be of computational burden. Therefore, an alternative solution, suboptimal but less expensive was adopted. It consists in taking $\rho^{(1)}=\rho^{(2)}=\rho^{(3)}=\rho$. Then 
$\rho$ is computed by rooting a fifth order polynomial. Regarding the complex case, the relaxation factor is defined by $\rho=r e^{j \theta}$ and then an iterative approach was proposed to estimate this latter [30]. Indeed, at each iteration estimating $\rho$ is achieved by alternating between two steps: a minimization of $\Psi$ with respect to (wrt) magnitude $r$ and a minimization of $\Psi$ wrt phase $\theta$. Following the same spirit and for $\rho^{(1)}=\rho^{(2)}=\cdots=\rho^{(q)}=\rho$, we generalize the ELS scheme proposed in [30] to arrays with order higher or equal to three. As far as $\boldsymbol{G}_{\boldsymbol{A}}^{(i)}$ is concerned, any type of search direction can be combined with the ELS procedure - we will make a particular choice in the computer result section.

Let $\Omega_{i}^{(r)}(q)$ be the $i$-th subset of $r$ elements taken from the set $\Omega(q)=\{1, \cdots, q\}$ of $q$ integers, sorted in an ascending way. The total number of combinations of $r$ elements taken among $q$ is given by $\left(\begin{array}{c}q \\ r\end{array}\right)=q ! /(r !(q-r) !)$. Inserting now the expression of $\boldsymbol{A}_{\text {new }}^{(i)}$ in (6) and using some matrix manipulations, $\Psi$ can be rewritten as:

$$
\Psi(\rho)=\left\|\boldsymbol{C}_{0}^{q}+\boldsymbol{C}_{1}^{q} \rho+\boldsymbol{C}_{2}^{q} \rho^{2}+\cdots+\boldsymbol{C}_{q}^{q} \rho^{q}\right\|_{F}^{2}
$$

where the $q+1$ matrices $\boldsymbol{C}_{n}^{q}$ are defined by:

$$
\boldsymbol{C}_{n}^{q}=\delta_{0, n} \boldsymbol{T}^{(q)}-\sum_{i=1}^{\left(\begin{array}{c}
q-1 \\
n
\end{array}\right)} \boldsymbol{A}^{(q)}\left(\bigotimes_{\omega \in \Omega_{i}^{(n)}(q-1)} \boldsymbol{B}^{(\omega)}\right)^{\top}-\sum_{k=1}^{\left(\begin{array}{c}
q-1 \\
n-1
\end{array}\right)} \boldsymbol{G}_{\boldsymbol{A}}^{(q)}\left(\bigotimes_{\omega \in \Omega_{k}^{(n-1)}(q-1)} \boldsymbol{B}^{(\omega)}\right)^{\mathrm{\top}}
$$

where $\delta_{0, n}=1$ if $n=0$ and 0 otherwise. In addition for $\omega \in\{1, \cdots, q-1\} \boldsymbol{B}^{(\omega)}$ is given by $\boldsymbol{B}^{(\omega)}=\boldsymbol{A}^{(\omega)}$ if $\omega \in \Omega_{i}^{(n)}(q-1)$ and $\boldsymbol{B}^{(\omega)}=\boldsymbol{G}_{\boldsymbol{A}}^{(\omega)}$ otherwise. Moreover, $\oslash$ denotes a succession of Khatri-Rao products and where $\Omega_{i}^{(0)}(q)=\Omega(q)$. Note that subscripts it and $i t-2$ have been omitted for the sake of convenience. Equation (7) then becomes:

$$
\Psi(\rho)=\|\widetilde{\boldsymbol{C}} \boldsymbol{u}\|_{F}^{2}=\boldsymbol{u}^{\mathrm{H}} \tilde{\boldsymbol{C}}^{\mathrm{H}} \tilde{\boldsymbol{C}} \boldsymbol{u}=\boldsymbol{u}^{\mathrm{H}} \boldsymbol{F} \boldsymbol{u}
$$

where $\widetilde{\boldsymbol{C}}=\left[\operatorname{vec}\left(\boldsymbol{C}_{q}^{q}\right), \operatorname{vec}\left(\boldsymbol{C}_{q-1}^{q}\right), \cdots, \operatorname{vec}\left(\boldsymbol{C}_{0}^{q}\right)\right]$ is a $\left(N_{1} N_{2} \cdots N_{q} \times q+1\right)$ matrix, $\boldsymbol{u}=$ $\left[\rho^{q}, \rho^{q-1}, \cdots, 1\right]^{\top}$ is a $(q+1)$-dimensional vector and matrix $\boldsymbol{F}$ is Hermitian. The minimization of $\Psi$ wrt $\rho=r e^{j \theta}$ is thus performed in an iterative way by alternating at each iteration between two steps [30]. The first step consists in minimizing $\Psi$ wrt $r$ while $\theta$ is fixed to its last estimate. To do so, $\Psi(9)$ can be written as a polynomial function of $r$ given by:

$$
\Psi(r)=\sum_{\substack{n=0 \\ 9}}^{2 q} d_{n} r^{n}
$$


where the coefficient $d_{n}$ is defined by:

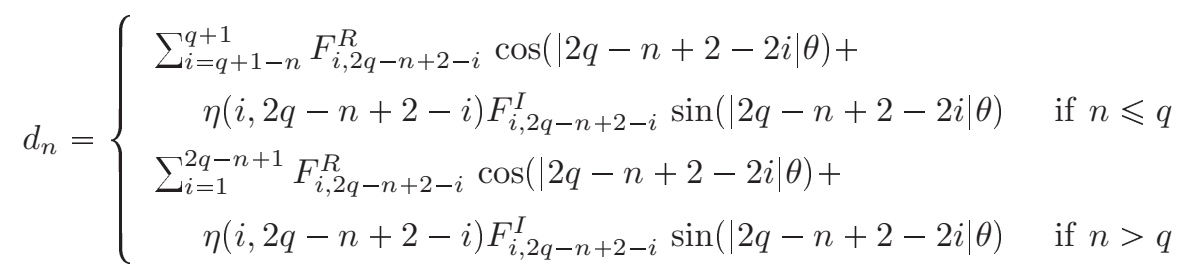

with $\eta(i, j)=1$ when $j \geqslant i$ and $\eta(i, j)=-1$ otherwise. In addition, $F_{i, j}^{R}$ and $F_{i, j}^{I}$ denote the real and the imaginary parts of the $(i, j)$-th entry of the Hermitian matrix $\boldsymbol{F}(9)$. Next, $r$ is updated by rooting a $(2 q-1)$-th polynomial given by:

$$
\frac{\partial \Psi(r)}{\partial r}=\sum_{n=0}^{2 q-1}(n+1) d_{n+1} r^{n}
$$

Fixing now $r$ to the root of (11), which minimizes (10), $\Psi$ is then minimized wrt $\theta$. To this end, $\Psi$ (9) has to be written as a function of $\theta$ :

$$
\Psi(\theta)=\sum_{i=0}^{q} F_{q+1-i, q+1-i}^{R} r^{2 i}+2 \sum_{n=1}^{q}\left(a_{n} \cos (n \theta)+b_{n} \sin (n \theta)\right)
$$

where:

$$
a_{n}=\sum_{m=1}^{q-n+1} F_{m, m+n}^{R} r^{2 q+2-n-2 m}, b_{n}=\sum_{m=1}^{q-n+1} F_{m, m+n}^{I} r^{2 q+2-n-2 m}
$$

Angle $\theta$ is then updated by computing the zeros of the following equation:

$$
\frac{\partial \Psi(\theta)}{\partial \theta}=2 \sum_{n=1}^{q} n\left(b_{n} \cos (n \theta)-a_{n} \sin (n \theta)\right)
$$

More precisely, first express both $\cos (n \theta)$ and $\sin (n \theta)$ as a function of $\cos (\theta)$ and $\sin (\theta)$ using the following equations:

$$
\begin{aligned}
& \cos (n \theta)=\sum_{\ell=0}^{\operatorname{Int}(n / 2)}(-1)^{\ell}\left(\begin{array}{c}
n \\
2 \ell
\end{array}\right) \cos (\theta)^{n-2 \ell} \sin (\theta)^{2 \ell} \\
& \sin (n \theta)=\sum_{\ell=0}^{\operatorname{Int}((n-1) / 2)}(-1)^{\ell}\left(\begin{array}{c}
n \\
2 \ell+1
\end{array}\right) \cos (\theta)^{n-1-2 \ell} \sin (\theta)^{2 \ell+1}
\end{aligned}
$$

where $\operatorname{Int}(n)$ denotes the integer part of $n$. These equations are derived using both Newton binomial and Moivre formulas as shown in appendix 9.1. Second resort to the change of variable, $t=\tan (\theta / 2)$, and next, use the following trigonometric statements:

$$
\cos (\theta)=\frac{1-t^{2}}{1+t^{2}} \quad{ }_{10} \sin (\theta)=\frac{2 t}{1+t^{2}}
$$


Then injecting equations (15), (16) into equation (14) and using the Newton binomial formula given in appendix, the update of $\theta$ is derived from the root of the following polynomial in variable $t$, which minimizes (12):

$$
\begin{aligned}
\frac{\partial \Psi(\theta)}{\partial \theta} & =2 \sum_{n=1}^{q} \sum_{\ell_{1}=0}^{\operatorname{Int}(n / 2)} \sum_{i=0}^{\operatorname{Int}(n / 2)-\ell_{1}} n b_{n}(-4)^{\ell_{1}}\left(\begin{array}{c}
n \\
2 \ell_{1}
\end{array}\right)\left(\begin{array}{c}
n-2 \ell_{1} \\
2 i
\end{array}\right) t^{2 \ell_{1}+4 i} \\
& -4 \sum_{n=1}^{q} \sum_{\ell_{2}=0}^{\operatorname{Int}((n-1) / 2)} \sum_{j=0}^{\operatorname{Int}((n-1) / 2)-\ell_{2}} n a_{n}(-4)^{\ell_{2}}\left(\begin{array}{c}
{ }_{2 \ell_{2}+1} \\
n
\end{array}\right)\left(\begin{array}{c}
n-1-2 \ell_{2} \\
2 j
\end{array}\right) t^{2 \ell_{2}+4 j+1}
\end{aligned}
$$

Example 3. Let $\mathcal{T}\left(\boldsymbol{A}, \boldsymbol{A}, \boldsymbol{A}, \boldsymbol{A}^{*}, \boldsymbol{A}^{*}, \boldsymbol{A}^{*}\right.$ ) be a Sixth Order (SixO) Hermitian positive semi-definite array (here $2 q=6$ ) defined according to equation (4) by:

$$
\mathcal{T}=\sum_{p=1}^{\operatorname{rk}(\mathcal{T})} \boldsymbol{a}_{p} \circ \boldsymbol{a}_{p} \circ \boldsymbol{a}_{p} \circ \boldsymbol{a}_{p}^{*} \circ \boldsymbol{a}_{p}^{*} \circ \boldsymbol{a}_{p}^{*}
$$

Then according to equation (7) we have:

$$
\Psi(\rho)=\left\|C_{0}^{6}+C_{1}^{6} \rho+C_{2}^{6} \rho^{2}+C_{3}^{6} \rho^{3}+C_{4}^{6} \rho^{4}+C_{5}^{6} \rho^{5}+C_{6}^{6} \rho^{6}\right\|_{F}^{2}
$$

where matrices $\boldsymbol{C}_{i}^{6}(1 \leqslant i \leqslant 6)$ are given by equation (8). Then computing the optimal relaxation factor $\rho=r e^{j \theta}$ is performed by alternating between a minimization of $\Psi$ wrt $r$ and a minimization wrt $\theta$. The minimization of $\Psi$ wrt $r$ is achieved by rooting the eleventh degree polynomial (11) where the coefficients $d_{n}(0 \leqslant n \leqslant 11)$ are given in appendix 9.2. Similarly, the minimization of $\Psi$ wrt $\theta$ is achieved by rooting the following twelfth degree polynomial in variable $t$ :

$$
\frac{\partial \Psi(\theta)}{\partial \theta}=\sum_{i=0}^{12} z_{i} t^{i}
$$

where $t=\tan (\theta / 2)$ and the expressions of coefficients $z_{k}(0 \leqslant k \leqslant 12)$ are given in the appendix 9.2.

3.2. CANDHAP (CAND of Hermitian positive semi-definite Arrays based on Procrustes problem)

A new ALS-like method, named CANDHAP, to fit the CAND of HO Hermitian positive semi-definite arrays is presented in this section. Using definitions 5-7, the unfolding 
matrix $\boldsymbol{T}=\operatorname{mat}_{1}(\mathcal{T})$ of a $2 q$-th $(q \geqslant 2)$ order Hermitian positive semi-definite array $\mathcal{T}$ has the following algebraic structure:

$$
\boldsymbol{T}=\boldsymbol{A}^{\oslash\lceil q / 2]} \oslash \boldsymbol{A}^{* \oslash[q / 2\rfloor} \boldsymbol{\Lambda}_{P}\left(\boldsymbol{A}^{\oslash[q / 2]} \oslash \boldsymbol{A}^{* \oslash \mid q / 2\rfloor}\right)^{\mathrm{H}}=\boldsymbol{A}_{q} \boldsymbol{\Lambda}_{P} \boldsymbol{A}_{q}^{\mathrm{H}}
$$

where $\boldsymbol{A}_{q}=\boldsymbol{A}^{\oslash[q / 2]} \oslash \boldsymbol{A}^{* \oslash\lfloor q / 2\rfloor}=\left[\boldsymbol{a}_{1}^{\otimes[q / 2]} \otimes \boldsymbol{a}_{1}^{* \otimes \mid q / 2\rfloor}, \cdots, \boldsymbol{a}_{P}^{\otimes[q / 2]} \otimes \boldsymbol{a}_{P}^{* \otimes[q / 2\rfloor}\right]$, where $\boldsymbol{a}_{p}$ is the $p$-th column vector of $\boldsymbol{A}$ and where $\boldsymbol{\Lambda}_{p}=\operatorname{diag}\left\{\left[\lambda_{1}, \cdots, \lambda_{P}\right]\right\}$ is the diagonal matrix built from the $P$ strictily positive values $\lambda_{p}$ involved in the CAND (4) of $\mathcal{T}$ (in the case when $\lambda_{p}<0$ for $1 \leqslant p \leqslant P$, matrix $-\boldsymbol{T}$ should be considered instead). Consequently, $\boldsymbol{T}$ is positive semi-definite, its square root exists and is defined as follows:

$$
\boldsymbol{T}^{1 / 2}=\boldsymbol{E} \boldsymbol{L}^{1 / 2}=\boldsymbol{A}_{q} \boldsymbol{\Lambda}_{P}^{1 / 2} \boldsymbol{B}^{\mathrm{H}}
$$

where $\boldsymbol{L}^{1 / 2}$ is the square root of the real-valued diagonal matrix of the $P$ non-zero eigenvalues of $\boldsymbol{T}, \boldsymbol{E}$ is the corresponding $\left(N^{q} \times P\right)$ unitary eigenmatrix, $\boldsymbol{\Lambda}_{P}^{1 / 2}$ is the square root of $\boldsymbol{\Lambda}$ and $\boldsymbol{B}$ is a $(P \times P)$ unitary matrix. The unitary property of $\boldsymbol{B}$ is derived from the fact that two square roots of the same matrix are equal up to a unitary matrix.

Let us now consider, for an arbitrary value of $q$, the $\left(P \times N^{q}\right)$ matrix $\left(\boldsymbol{T}^{1 / 2}\right)^{\top}$

Then we can write:

$$
\begin{aligned}
\left(\boldsymbol{T}^{1 / 2}\right)^{\top} & =\boldsymbol{B}^{*} \boldsymbol{\Lambda}_{P}^{1 / 2} \boldsymbol{A}_{q}^{\top}=\boldsymbol{B}^{*} \boldsymbol{\Lambda}_{P}^{1 / 2}\left(\boldsymbol{A} \oslash \boldsymbol{W}_{q-1}\right)^{\top} \\
& =\boldsymbol{B}^{*}\left(\boldsymbol{A} \boldsymbol{\Lambda}_{P}^{1 / 2} \oslash \boldsymbol{W}_{q-1}\right)^{\top}=\boldsymbol{B}^{*}\left(\boldsymbol{D} \oslash \boldsymbol{W}_{q-1}\right)^{\top}=\operatorname{mat}_{2}^{(2)}(\tilde{\mathcal{T}})
\end{aligned}
$$

where:

$$
\boldsymbol{W}_{q-1}= \begin{cases}\boldsymbol{A}_{q-1} & \text { if } q \text { is odd } \\ \boldsymbol{\Pi} \boldsymbol{A}_{q-1}^{*} & \text { if } q \text { is even }\end{cases}
$$

where $\Pi$ is a permutation matrix defined as:

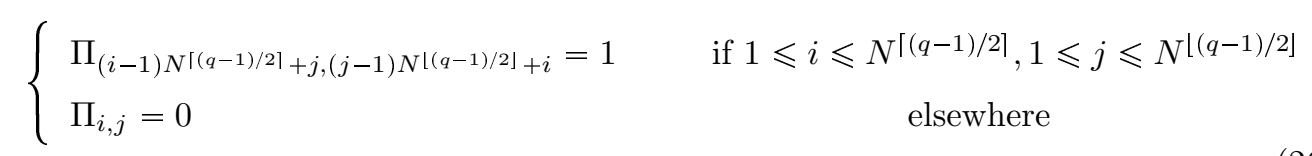

According to equation $(24),\left(\boldsymbol{T}^{1 / 2}\right)^{\top}$ can be seen as the unfolding matrix associated to the second direction of a third order array, named $\widetilde{\mathcal{T}}\left(\boldsymbol{W}_{q-1}, \boldsymbol{B}^{*}, \boldsymbol{D}\right)$, of size $\left(N^{q-1} \times P \times N\right)$ and whose loading matrices are $\boldsymbol{W}_{q-1}, \boldsymbol{B}^{*}$ and $\boldsymbol{D}=\boldsymbol{A} \boldsymbol{\Lambda}_{P}^{1 / 2}$. According to definition 4 the CAND of $\tilde{\mathcal{T}}$ can then be written as:

$$
\tilde{\mathcal{T}}=\sum_{p=1}^{P} \boldsymbol{w}_{q-1, p} \circ \boldsymbol{b}_{p}^{*} \circ \boldsymbol{d}_{p}
$$


where $\boldsymbol{w}_{q-1, p}, \boldsymbol{b}_{p}$ and $\boldsymbol{d}_{p}$ are the $p$-th columns of matrices $\boldsymbol{W}_{q-1}, \boldsymbol{B}$ and $\boldsymbol{D}$, respectively. According to equation (22), identifying matrix $\boldsymbol{A}_{q}$ is immediate once the unitary matrix $\boldsymbol{B}$ is well-estimated. Since $\boldsymbol{B}$ is a loading matrix of $\tilde{\mathcal{T}}(27)$, its estimation is then possible by fitting the CAND of $\tilde{\mathcal{T}}$. Note that each frontal slice of $\tilde{\mathcal{T}}$ has the following algebraic structure:

$$
\forall 1 \leqslant n \leqslant N, \quad \tilde{\mathcal{T}}(:,:, n)=\boldsymbol{W}_{q-1} \operatorname{diag}\{\boldsymbol{D}(n,:)\} \boldsymbol{B}^{\mathrm{H}}
$$

where $\boldsymbol{D}(n,:)$ denotes the $n$-th row of $\boldsymbol{D}$. Since $\boldsymbol{B}$ is unitary, then equation (28) can be seen as a generalized version of the Procrustes problem defined by:

Problem 1. Given $N$ couples of matrices $\boldsymbol{G}_{n}$ and $\boldsymbol{F}_{n}$ of the same size, find a unitary matrix $\boldsymbol{B}$ that minimizes the following least squares criterion:

$$
\Psi(\boldsymbol{B})=\sum_{n=1}^{N}\left\|\boldsymbol{G}_{n}-\boldsymbol{F}_{n} \boldsymbol{B}^{\mathrm{H}}\right\|_{F}^{2}
$$

The solution of this problem is well-known to be $\boldsymbol{B}=\boldsymbol{V} \boldsymbol{U}^{\mathrm{H}}[32$, chapter 12] where $\boldsymbol{U}$ and $\boldsymbol{V}$ are the matrices of the left and right singular vectors of matrix $\sum_{n} \boldsymbol{F}_{n}^{H} \boldsymbol{G}_{n}$, respectively. Nevertheless, such a solution is not directly applicable in our case since according to equation (28) neither $\boldsymbol{W}_{q-1}$ nor $\boldsymbol{D}$ are known. Thus, resorting to an alternating optimization scheme is suitable in such a case in order to estimate the three loading matrices $\boldsymbol{W}_{q-1}, \boldsymbol{B}$ and $\boldsymbol{D}$. Author in [48, pages 62-63] proposed a similar idea in order to canonically decompose a real-valued three-way array with one orthogonal loading matrix. It is noteworthy that no computer result was given in [48] to assess the behavior of such an approach. But instead of alternating between three optimization procedures, we propose to alternate between only two optimization procedures based on the following proposition:

Proposition 1. The $p$-th vertical slice of $\mathcal{T}_{1}\left(\boldsymbol{W}_{q-1}, \boldsymbol{B}^{*}, \boldsymbol{D}\right)=\tilde{\mathcal{T}} \times_{2} \boldsymbol{B}^{\mathrm{T}}$ is a rank-1 matrix.

Proof. According to equation (24) and since $\boldsymbol{B}$ is unitary, we have:

$$
\mathcal{T}_{1}=\tilde{\mathcal{T}} \times_{2} \boldsymbol{B}^{\top}=\boldsymbol{B}^{\top} \operatorname{mat}_{2}^{(2)}(\tilde{\mathcal{T}})=\mathbf{I}_{P}\left(\boldsymbol{D} \oslash \boldsymbol{W}_{q-1}\right)^{\top}=\operatorname{mat}_{2}^{(2)}\left(\mathcal{T}_{1}\right)
$$


Then $\mathcal{T}_{1}\left(\boldsymbol{W}_{q-1}, \mathbf{I}_{P}, \boldsymbol{D}\right)$ is a third order array whose loading matrix associated to its second mode is the identity and consequently its $p$-th vertical slice is given by:

$$
\mathcal{T}_{1}(:, p,:)=\boldsymbol{W}_{q-1} \operatorname{diag}\left\{\mathbf{I}_{P}(p,:)\right\} \boldsymbol{D}^{\top}=\boldsymbol{w}_{q-1, p} \boldsymbol{d}_{p}^{\top}=\boldsymbol{w}_{q-1, p} \circ \boldsymbol{d}_{p}
$$

which is, according to definition 1 , a rank-1 matrix.

As a result, using the last estimate of $\boldsymbol{B}$, updating both vectors $\boldsymbol{w}_{q-1, p}$ and $\boldsymbol{d}_{p}$, at each iteration, is possible by computing the left and the conjugate of the right singular vectors associated to the largest singular value of the $p$-th matrix $\mathcal{T}_{1}(:, p,:)$, respectively. Next, an update of $\boldsymbol{B}$ is done using the solution of problem 1. That is to say, using the SVD of the $(P \times P)$ matrix $\sum_{n=1}^{N}(\operatorname{diag}\{\boldsymbol{D}(n,:)\})^{*} \boldsymbol{W}_{q-1}^{\mathrm{H}} \tilde{\mathcal{T}}(:,:, n)$. All previous steps are repeated until the convergence of the algorithm. Algorithm 1 summarizes the proposed procedure, named CANDHAP1, to fit the CAND of $\tilde{\mathcal{T}}(27)$. The algorithm stops when the error between the left and the right hand sides of (27) exhibits, between two iterations, a change smaller than a predefined threshold. Once the convergence is attained, the $P$ factors $\boldsymbol{a}_{p}$ are computed either by looking for the best rank-1 approximation of $P q$-th order arrays with the HOPI method [37] or by repeating $P$ times the joint diagonalization of $N^{q-2}$ rank-1 matrices with for instance the diagonalization schemes described in $[49,50,51]$. Indeed, consider a rank-1 $q$-way array $\mathcal{A}=\boldsymbol{y}^{(1)} \circ \cdots \circ \boldsymbol{y}^{(q)}$, then, at each iteration of the HOPI method, vector $\boldsymbol{y}_{i t+1}^{(i)}$ is estimated in the following way:

$$
\boldsymbol{y}_{i t+1}^{(i)}=\mathcal{A} \times{ }_{1}\left(\boldsymbol{y}_{i t+1}^{(1)}\right)^{\mathrm{T}} \times_{2} \cdots \times_{i-1}\left(\boldsymbol{y}_{i t+1}^{(i-1)}\right)^{\mathrm{T}} \times_{i+1}\left(\boldsymbol{y}_{i t}^{(i+1)}\right)^{\mathrm{T}} \times_{i+2} \cdots \times_{q}\left(\boldsymbol{y}_{i t}^{(q)}\right)^{\mathrm{T}}
$$

In our case, the $p$-th factor $\boldsymbol{a}_{p}$ is estimated by looking for the best rank-1 approximation of the $p$-th rank-1 $q$-th order array $\mathcal{A}_{p} \in \mathbb{C}^{N \times N \times \cdots \times N}$ defined as $\mathcal{A}_{p}=\operatorname{unvec}\left(\boldsymbol{r}_{p}\right)$ where $\boldsymbol{r}_{p}$ is the $p$-th column of $\boldsymbol{R}=\boldsymbol{T}^{1 / 2} \boldsymbol{B}$. As far as the semi-algebraic scheme is considered, the $p$-th factor $\boldsymbol{a}_{p}$ is computed as the joint diagonalizer of $N^{q-2}$ rank-1 matrices built from the $p$-th column of the $\left(N^{q} \times P\right)$ matrix $\boldsymbol{R}$. Indeed for each column $\boldsymbol{r}_{p}$ of $\boldsymbol{R}$, first $N^{q-2} N^{2}$-dimensional vectors $\boldsymbol{z}_{p}^{m}\left(1 \leqslant m \leqslant N^{q-2}\right)$ are extracted where each vector $\boldsymbol{z}_{p}^{m}$ is defined such as in [13]. Then each vector $z_{p}^{m}$ is remodeled as a matrix of size $(N \times N)$, and finally the set of the resulting $N^{q-2}$ matrices is jointly diagonalized. This joint diagonalization can be performed by means of Jacobi-like algorithms such as the JAD one [49]. Algorithm 2, named CANDHAP2, summarizes the global proposed algorithm to fit the CAND of Hermitian positive semi-definite array $\mathcal{T}(4)$. 
\begin{tabular}{l}
\hline Algorithm 1 (CANDHAP1): Find the CAND of the rank- $P$ 3-way array \\
$\tilde{\mathcal{T}}\left(\boldsymbol{W}_{q-1}, \boldsymbol{B}^{*}, \boldsymbol{D}\right) \in \mathbb{C}^{N^{q-1} \times P \times N}(27)$ with $\boldsymbol{B}$ unitary. \\
\hline
\end{tabular}

Step1 Build $\boldsymbol{T}=\operatorname{mat}_{2}^{(2)}(\tilde{\mathcal{T}})$.

Step2 Repeat until the convergence: i) $\mathcal{T}_{1}=\tilde{\mathcal{T}} \times_{2} \boldsymbol{B}^{\top} \Rightarrow \forall 1 \leqslant p \leqslant P, \quad \mathcal{T}_{1}(:, p,:)=$ $\boldsymbol{w}_{q-1, p} \boldsymbol{d}_{p}^{\top}$, ii) $\operatorname{SVD}\left(\mathcal{T}_{1}(:, p,:)\right)=\boldsymbol{U}_{p} \boldsymbol{\Sigma}_{p} \boldsymbol{V}_{p}^{\mathrm{H}} \Rightarrow$ take both vectors $\boldsymbol{w}_{q-1, p}$ and $\boldsymbol{d}_{p}$ as the left and the right singular vectors associated to the largest singular value in $\boldsymbol{\Sigma}_{p}$, respectively and iii) Compute the SVD: $\sum_{n=1}^{N}(\operatorname{diag}\{\boldsymbol{D}(n ;:)\})^{*} \boldsymbol{W}_{q-1}^{\mathrm{H}} \widetilde{\mathcal{T}}(:,:, n)=$ $\boldsymbol{U} \boldsymbol{\Sigma} \boldsymbol{V}^{\mathrm{H}}$. Then, update $\boldsymbol{B}$ as $\boldsymbol{B}=\boldsymbol{U} \boldsymbol{V}^{\mathrm{H}}$.

$\overline{\text { Algorithm } 2 \text { (CANDHAP2) Find the CAND of 2q-th order }(q>2) \text { Hermitian positive }}$ semi-definite array $\mathcal{T}(4)$.

Step1 Build $\boldsymbol{T}=\operatorname{mat}_{1}(\mathcal{T})$ and compute $P=\operatorname{rank}(\boldsymbol{T})$.

Step2 Compute a square root, $\boldsymbol{T}^{1 / 2}$, of $\boldsymbol{T}$ (see equation (22)).

Step3 Build $\tilde{\mathcal{T}}\left(\boldsymbol{W}_{q-1}, \boldsymbol{B}^{*}, \boldsymbol{A}\right)(27): \tilde{\mathcal{T}}=\operatorname{unmat}_{2}^{(2)}\left(\left(\boldsymbol{T}^{1 / 2}\right)^{\top}\right)$.

Step4 Find the CAND of $\tilde{\mathcal{T}}$ using the CANDHAP1 approach.

Step5 From $\boldsymbol{B}$ estimated using step 5, build $\boldsymbol{A}_{q}=\boldsymbol{T}^{1 / 2} \boldsymbol{B}=\left[\boldsymbol{a}_{q, 1}, \cdots, \boldsymbol{a}_{q, P}\right]$.

Step6 Build the $P q$-th order rank-1 arrays $\mathcal{A}_{p}=\operatorname{unvec}\left(\boldsymbol{a}_{q, p}\right)$.

Step7 Compute the best rank-1 approximation of each array $\mathcal{A}_{p}$ using either the HOPI method [37] or the approach based on joint matrix diagonalization [13]. 
It is noteworthy that two different ways can be used to initialize the HOPI method [37] when processing the $p$-th array $\mathcal{A}_{p}$. The first one consists of using the truncated HOSVD [37]. The second one is to take the $p$-th column of $\boldsymbol{D}=\left[\boldsymbol{d}_{1}, \cdots, \boldsymbol{d}_{P}\right](31)$ as a start guess. The latter solution is valid since the loading matrix $\boldsymbol{D}$ associated to the third mode of $\tilde{\mathcal{T}}(27)$ is equal, up to a diagonal matrix, to $\boldsymbol{A}=\left[\boldsymbol{a}_{1}, \cdots, \boldsymbol{a}_{P}\right]$. Note also that the CAND of $\tilde{\mathcal{T}}$ coud be computed without any orthogonality constraint on matrix B. But in practice, the CAND analysis of a third order array is complicated by the occurrence of "degeneracies" [52] while running a CAND algorithm. In such cases, the CAND criterion function decreases very slowly, the estimated loading matrices diverge towards an infimum that is not a minimum (individual terms go to infinity but they more or less compensate, resulting in an ever improving fit), and the loading matrices become nearly rank deficient. However, it was shown in [53] that degeneracies can be avoided by imposing orthogonality restrictions on at least one of the three loading matrices. Hence our choice to use the orthogonality of $\boldsymbol{B}$ in the optimization procedure.

\section{Uniqueness}

This section is devoted to study under which conditions on $P$, the solution proposed to fit the CAND of a $2 q$-th order $(q \geqslant 2)$ array of Hermitian symmetry, is unique up to the inherent indeterminacies of the CAND (e.g., scaling and permutation). Then, a generic $\mathrm{HO}$ array with these previous properties and whose rank satisfies this condition, has a CAND that is unique and comprises components that can be computed by means of the CANDHAP2 algorithm. Since fitting the CAND of $\mathcal{T}(4)$ relies essentially on fitting the CAND of the third order array $\tilde{\mathcal{T}}\left(\boldsymbol{W}_{q-1}, \boldsymbol{B}^{*}, \boldsymbol{D}\right) \in \mathbb{C}^{N^{q-1} \times P \times N}(27)$ with $\boldsymbol{B}$ being unitary, the uniqueness condition will be basically derived from the CANDHAP1 algorithm.

Assume that matrix $\boldsymbol{A}$ is randomly sampled from an $N P$-dimensional continuous distribution. Now, for $q$ odd, matrix $\boldsymbol{D} \oslash \boldsymbol{W}_{q-1}$ is equal to $\boldsymbol{A} \oslash \boldsymbol{A}_{q-1}$ up to a diagonal matrix $\boldsymbol{\Lambda}_{P}^{1 / 2} \oslash \mathbf{I}_{N^{q-1}}$. Note that multiplying a matrix by an invertible diagonal one does not affect its rank. Now, we can write $\boldsymbol{A} \oslash \boldsymbol{A}_{q-1}=\boldsymbol{A}^{\oslash[q / 2\rceil} \oslash \boldsymbol{A}^{\oslash *\lfloor q / 2\rfloor}=$ $\left[\boldsymbol{a}_{1}^{\otimes[q / 2]} \otimes \boldsymbol{a}_{1}^{* \otimes[q / 2\rfloor}, \cdots, \boldsymbol{a}_{P}^{\otimes[q / 2]} \otimes \boldsymbol{a}_{P}^{* \otimes[q / 2\rfloor}\right]$. Due to the symmetry property of matrix $\boldsymbol{A}^{\oslash\lceil q / 2\rceil}\left(\boldsymbol{A}^{* \oslash\lceil q / 2\rfloor}\right.$ respectively), some rows appear $\lceil q / 2\rceil(\lfloor q / 2\rfloor$ respectively) times. Then, 
keeping only one of the repeated rows is required when evaluating the rank of this matrix. Let $\underline{\boldsymbol{A}}_{\{q / 2\rceil}$ and $\underline{\boldsymbol{A}}_{\lfloor q / 2\rfloor}^{*}$ be two matrices of size $\left(\mathcal{N}_{\lceil q / 2\rceil} \times P\right)$ and $\left(\mathcal{N}_{\lfloor q / 2\rfloor} \times P\right)$, respectively, and obtained once redundancies in $\boldsymbol{A}^{\oslash[q / 2]}$ and $\boldsymbol{A}^{* \oslash \mid q / 2]}$ are removed. The numbers $\mathcal{N}_{[q / 2\rceil}$ and $\mathcal{N}_{[q / 2]}$ of the distinct rows are computed using the combinatorial analysis with repetition. More precisely, $\mathcal{N}_{\ell}$ is computed by looking for the combinations of $\ell$ elements drawing from $N$ distinct ones without taking into account their apparition order. Hence, $\mathcal{N}_{\ell}=\left(\begin{array}{c}N+\ell-1 \\ \ell\end{array}\right)$. Then, since no redundancy can be now found in $\underline{\boldsymbol{A}}_{\lceil q / 2\rceil} \oslash \underline{\boldsymbol{A}}_{\lfloor q / 2\rfloor}^{*}$ and since $\boldsymbol{B}$ has a full column rank, the CAND of $\mathcal{T}$ is essentially unique with probability 1 if $\left(\underline{\boldsymbol{A}}_{\lceil q / 2\rceil} \oslash \underline{\boldsymbol{A}}_{[q / 2]}^{*}\right)$ has a full column rank [54, Theorem 1]. According to the results of [54], $\left(\underline{\boldsymbol{A}}_{\lceil q / 2\rceil} \oslash \underline{\boldsymbol{A}}_{[q / 2]}^{*}\right)$ is full column rank if the following inequality holds.

$$
\frac{P(P-1)}{2} \leqslant \frac{\mathcal{N}_{\lceil q / 2\rceil}\left(\mathcal{N}_{\lceil q / 2\rceil}-1\right) \mathcal{N}_{\lfloor q / 2]}\left(\mathcal{N}_{\lfloor q / 2\rfloor}-1\right)}{4}
$$

When $q$ is even, matrix $\boldsymbol{D} \oslash \boldsymbol{W}_{q-1}$ is equal to $\boldsymbol{A} \oslash \boldsymbol{A}_{q-1}^{*}$ up to matrix $\boldsymbol{\Lambda}_{P}^{1 / 2} \oslash \boldsymbol{\Pi}$ which is full column rank. Then similarly to the case when $q$ is odd, the same inequality in equation (33) can be found but with $\lfloor q / 2\rfloor=\lceil q / 2\rceil=q / 2$.

\section{Numerical complexity}

In this section we tackle the numerical complexity of the generalized ELSALS procedure and that of several variants of CANDHAP2. These different versions share steps 1 to 6 but they differ in the way they compute the best rank-1 approximation (see step 7). Therefore, the numerical complexity of step 7 is computed first while the numerical complexity of the left steps are computed thereafter. The numerical complexity is expressed hereafter in complex flops. A flop corresponds to a multiplication followed by an addition. In practice, the numerical complexity is assimilated to the number of multiplications, since multiplications are more expansive than additions.

Based on the implementation presented in section 3.1, the ELSALS scheme requires $\left(6\left(P\left(N^{5}+N^{4}+N^{3}+N^{2}\right)+11 P^{3} / 3+P^{2}+P+8 N^{5} P^{2}+P N^{6}\right)+8\left(P N^{2}+P N^{3}\right)+\right.$ $\left.8 P N^{4}+10 P N^{5}+12 P N^{6}+49 N^{6}+537 i t_{2}\right) i t_{1}$ flops where $i t_{1}$ and $i t_{2}$ denote the number of iterations used by ALS and that used to estimate the relaxation factor of the ELS step, respectively. 
As far as CANDHAP2 is concerned, two techniques are considered in order to compute a best rank-1 approximation (see step 7). These techniques, whose convergence is linear, are the HOPI method [37] and the simultaneous matrix diagonalization using the JAD algorithm [49]. Each iteration of the HOPI method, when a $q$-th order array is considered, requires the estimation of $q$ vectors with $q-1$ matrix multiplications for each. The numerical complexity is then equal to $q\left(N^{q}+N^{q-1}+\cdots+N^{2}+N\right) t_{1}$ complex flops where $t_{1}$ denotes the number of used iterations. This amount will be increased by $q\left(2 N^{q+1}-\right.$ $2 N^{3} / 3+5 N-1 / 3$ ) complex flops when a truncated HOSVD is used as an initialization procedure. As far as the numerical complexity of the JAD algorithm [49] is concerned, it is equal to $P I N(N-1)\left(4 N^{q-1}+17 N^{q-2}+4 N+75\right) / 2$ complex flops since there exists $N^{q-2}$ Hermitian matrices per $q$-th order rank- 1 array to be jointly diagonalized where $I$ denotes the number of executed sweeps.

Regarding the numerical complexity of the other steps of CANDHAP2, it relies essentially on the iterative approach CANDHAP1 (i.e. step 4). Each iteration in the CANDHAP1 algorithm requires i) $P^{2} N^{q}$ complex flops to build the third order array $\mathcal{T}_{1}\left(\boldsymbol{A}_{q-1}, \mathbf{I}_{P}, \boldsymbol{D}\right)(31)$, ii) $P\left(2 N^{q+1}-2 N^{3} / 3+5 N^{q-1}+5 N-2 / 3\right)$ complex flops to estimate the dominant right and left singular vector of the $P$ vertical slices of $\mathcal{T}_{1}$ and iii) $2 P^{2} N^{q}+35 P^{3} / 3$ complex flops to compute the unitary matrix $\boldsymbol{B}$ by solving the generalized Procrustes problem. As a result the total numerical complexity of CANDHAP1 is equal to $\left(3 N^{q} P^{2}+2 P N^{q+1}+5 P N^{q-1}-2 P N^{3} / 3+5 P N+35 P^{3} / 3\right) t_{2}$ complex flops where $t_{2}$ stands for the used number of iterations. This amount gives along with the computation of both $\boldsymbol{T}^{1 / 2}$ (i.e. step 2) and the best rank-1 approximation (i.e. step 7), say the numerical complexity of the whole CANDHAP2 algorithm. That is $4 N^{3 q} / 3+2 N^{q} P^{2}+$ $\left(3 N^{q} P^{2}+2 P N^{q+1}+5 P N^{q-1}-2 P N^{3} / 3+5 P N+35 P^{3} / 3\right) t_{2}+C o m p(\operatorname{Step} 7)$ complex flops where $\operatorname{Comp}(\mathrm{Step} 7)$ stands for the complexity of step 7. The latter depends on the used rank-1 approximation technique.

\section{Simulation results}

A first analysis is presented in terms of convergence speed aiming on the one hand at comparing the ALS algorithm with our extended ELSALS technique, and on the other hand at assessing the behavior of the CANDHAP1 method. Next, the performance 
of the proposed ELSALS and CANDHAP2 algorithms is studied as a function of the Signal-to-Noise Ratio (SNR) in terms of both decomposition accuracy and numerical complexity. Note that when the convergence speed analysis is considered, it is meaningful to evaluate only the CANDHAP1 approach instead of the global CANDHAP2 technique since the iterative part of CANDHAP1 is the core of CANDHAP2. It is worth mentioning that evaluating the convergence speed of the classical ALS algorithm with and without the ELS step may be not original in its spirit since several studies have been conducted to show how ELS considerably speeds up the convergence of ALS in the presence of ill-conditioned third order arrays [27, 30]. However, the novelty in our study relies on evaluating the ELSALS behavior when processing complex arrays with order higher than three such as SixO arrays, which, shows the interest of using our generalized ELS procedure.

More particularly, a SixO rank-5 Hermitian positive semi-definite array $\mathcal{T}$ of dimensions three is considered throughout this section and defined according to equation (18). As pointed out previously, this kind of arrays is often encountered in practice such as in radiocommunications when resorting to HOS in order to solve the BUMI problem [13, 36] (see section 7). In this context, $\mathcal{T}(18)$ represents the SixO cumulant array obtained from the available observations acquired through $N$ sensors. Then, the $p$-th rank- 1 term in (18) denotes the contribution of the $p$-th user to these observations. Therefore and to make our experiments somehow realistic, the loading matrix $\boldsymbol{A}=\left[\boldsymbol{a}_{1}, \cdots, \boldsymbol{a}_{P}\right]$, is generated such that its $p$-th column $\boldsymbol{a}_{p}$ models the transfer between the $p$-th user and a Uniform Circular Array (UCA) of $N=3$ sensors. Thus the $n$-th element, $A_{n, p}$, of $\boldsymbol{a}_{p}$ is given by [13]:

$$
A_{n, p}=\exp \left\{j 2 \pi\left[x_{n} \cos \left(\gamma_{p}\right) \cos \left(\phi_{p}\right)+y_{n} \cos \left(\gamma_{p}\right) \sin \left(\phi_{p}\right)\right]\right\}
$$

where $x_{n}=(R / \lambda) \cos (2 \pi(n-1) / N), y_{n}=(R / \lambda) \sin (2 \pi(n-1) / N)$ and $R / \lambda=0.55$. Regarding the Directions of Arrival (DOAs) $\gamma_{p}$ and $\phi_{p}$, the $\gamma_{p}$ 's are chosen equal to zero while the $\phi_{p}$ 's are randomly generated at each realization such that the angular spacing $\Delta \phi_{i, i+1}$ between two successive angles is fixed to a predefined value $\Delta \phi$. All results reported here are averaged over 200 trials. Note that the numerical complexity required to compute the SixO cumulant array, which is of order $N^{6} / 72$ flops, was taken into account in all the numerical complexity expressions. 


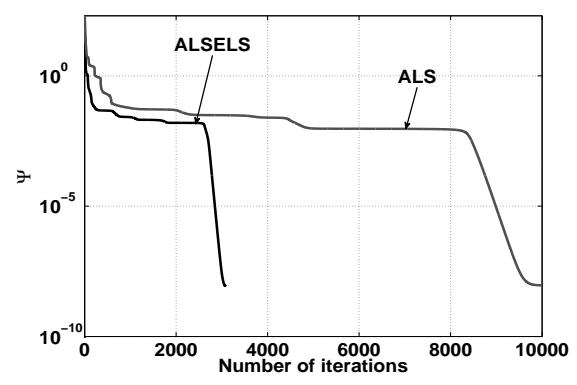

Figure 1: Convergence speed of ALS and ELSALS applied to a SixO Hermitian positive semi-definite array with ill-conditioned loading matrices.

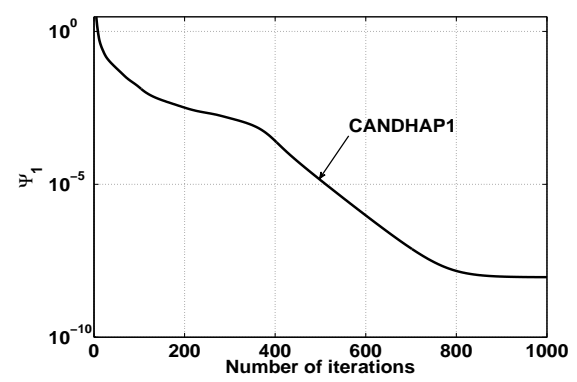

Figure 2: Convergence speed of CANDHAP1 applied to a SixO Hermitian positive semi-definite array with ill-conditioned loading matrices.

\subsection{Convergence speed analysis}

As mentioned previously, we compare here the convergence speed of ALS with that of the generalized ELSALS method. This study is performed by making close some factors $\boldsymbol{a}_{p}$ of the loading matrix $\boldsymbol{A}$. Results are reported in the noise-free case when the angular spacing between two successive DOAs is relatively small and equal to 25 degrees. In this case, a bottleneck-like situation is expected which will affect the convergence speed of the considered algorithms. Recall that a bottleneck is defined when the collinearity between some factors is present in two or more directions of the considered HO array [27]. Figure 1 shows the evolution of the cost function $\Psi$ defined by:

$$
\Psi(\boldsymbol{A})=\left\|\operatorname{mat}_{2}^{(6)}(\mathcal{T})-\boldsymbol{A}^{*}\left(\boldsymbol{A} \oslash \boldsymbol{A} \oslash \boldsymbol{A} \oslash \boldsymbol{A}^{*} \oslash \boldsymbol{A}^{*}\right)^{\top}\right\|_{F}^{2}
$$

as a function of the number of iterations. The ALS and ELSALS algorithms stop when $\Psi$ exhibits a value less or equal to $10^{-8}$. In addition, the six research directions $\boldsymbol{G}_{\boldsymbol{A}}^{(i)}$ in equation (6) are defined here as $\boldsymbol{G}_{\boldsymbol{A}}^{(i)}=\boldsymbol{A}_{i t-1}^{(i)}-\boldsymbol{A}_{i t-2}^{(i)}$. Regarding the initialization, ten random points were generated and tested over ten iterations of the ALS algorithm. Then we retained among these ten points the one which fits better the CAND of $\mathcal{T}$ (18). As expected, in the presence of close factors, ALS shows some slowness since it takes approximatively ten thousand iterations to attain the optimal solution, as displayed in figure 1. This is due to some convergence cycles wherein ALS spends more iterations to reach the final solution. On the other hand, figure 1 shows that the use of the SixO ELS step increases considerably the convergence speed of the ALS algorithm since it helps in 
attaining the final solution with only three thousand iterations. Note here that despite the fact that the convergence speed of the ELSALS algorithm is considerably higher than the one of the ALS approach, the numerical complexity of the latter is approximately four times lower than the one of the former.

Let us now evaluate the convergence speed of CANDHAP1 algorithms when it is used as part of the CANDHAP2 technique. Indeed, as described in section 3.2, CANDHAP1 can be used to decompose the special 3-way array $\tilde{\mathcal{T}}$ built from $\mathcal{T}$ (18) according to steps 1-3 of the CANDHAP2 algorithm. Then, following both equations (27) and (25), the CAND of $\tilde{\mathcal{T}}$ is computed by minimizing the following cost function:

$$
\Psi_{1}\left(\boldsymbol{A}_{2}, \boldsymbol{B}, \boldsymbol{D}\right)=\left\|\tilde{\mathcal{T}}-\sum_{p=1}^{5} \boldsymbol{a}_{2, p} \circ \boldsymbol{b}_{p}^{*} \circ \boldsymbol{d}_{p}\right\|_{F}^{2}
$$

under the constraint $\boldsymbol{B}^{\mathrm{H}} \boldsymbol{B}=\mathbf{I}_{P}$, where $\boldsymbol{a}_{2, p}=\boldsymbol{a}_{p} \otimes \boldsymbol{a}_{p}^{*}$ and $\boldsymbol{b}_{p}, \boldsymbol{d}_{p}$ are the $p$-th column of matrices $\boldsymbol{B}$ and $\boldsymbol{D}$, respectively (see equations (23)-(24)). Similarly to the previous experiment, CANDHAP1 stops when $\Psi_{1}$ exhibits a value less or equal to $10^{-8}$, with initial guess obtained such as in the previous experiment. Figure 2 shows how the proposed CANDHAP1 method succeeds in attaining the optimal solution within a reasonable number of iterations even in the presence of close factors.

\subsection{Influence of the $S N R$}

The goal of this section is to evaluate the performance of the proposed CANDHAP2 algorithm compared to ELSALS. This is performed in terms of decomposition accuracy and numerical complexity as a function of SNR. Therefore, a noisy SixO array $\mathcal{Y}$ defined as:

$$
\mathcal{Y}=\frac{\mathcal{T}}{\|\mathcal{T}\|_{F}}+\sigma_{\nu} \frac{\mathcal{N}}{\|\mathcal{N}\|_{F}}
$$

is considered where $\mathcal{T}$ is generated such as described in the previous experiments, $\mathcal{N}$ is a $\mathrm{SixO}$ array whose elements are drawn from a zero-mean normal distribution with a standard deviation equal to one and $\sigma_{\nu}$ is a scalar that controls the noise level. The performance criterion is taken as the mean relative error defined as:

$$
e=\mathrm{E}\left[\frac{\|\boldsymbol{A}-\hat{\boldsymbol{A}}\|_{F}}{\|\boldsymbol{A}\|_{F}}\right]
$$




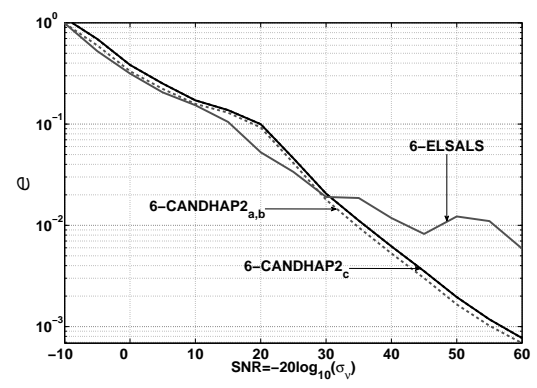

Figure 3: Influence of SNR for the CAND of a SixO Hermitian positive semi-definite array with ill-conditioned loading matrices.

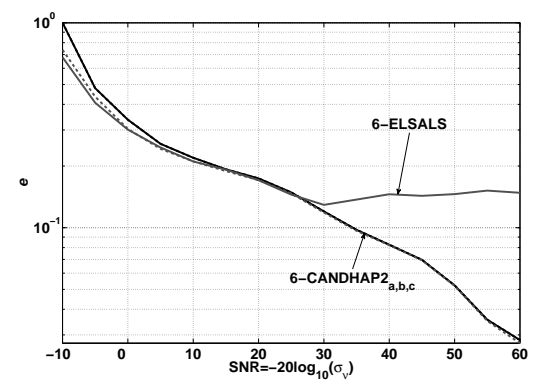

Figure 4: Influence of SNR for the CAND of a SixO Hermitian positive semi-definite array with very ill-conditioned loading matrices.

where $\hat{\boldsymbol{A}}$ represents the optimally ordered and scaled estimate of $\boldsymbol{A}$. Algorithms in this experiment stop when the model error between two successive iterations exhibits a change less than a predefined threshold $\left(\epsilon=10^{-9}\right)$. As pointed out previously, three variants of the proposed algorithm can be distinguished. All of them share steps 1-7 of the CANDHAP2 algorithm while they differ in the way they identify the $P$ vectors $\boldsymbol{a}_{p}$. Therefore, the acronym CANDHAP $2_{a}$ will refer to the case when the HOPI method with a truncated HOSVD for initialization is used [37]. The acronym CANDHAP $2_{b}$ will refer to the case when the HOPI method [37] is initialized with an estimate of $\boldsymbol{A}$ resulting from step 4 (i.e. CANDHAP1 method) and the acronym CANDHAP $2_{c}$ will denote the case when the JAD scheme [49] for joint matrix diagonalization is employed.

\subsubsection{Accuracy study}

The first experiment conducted here is the case where $\Delta \phi=25^{\circ}$. Figure 3 shows the accuracy of the CANDHAP2 method as a function of SNR and compared to the ELSALS algorithm. For low SNR values, all considered methods here show a comparable performance while the estimation quality of the CANDHAP2 approaches becomes higher than the one of the ELSALS algorithm beyond SNR of $30 \mathrm{~dB}$.

Let us now evaluate the aforementioned methods in a more difficult situation, i.e. $\Delta \phi=$ $15^{\circ}$. In this case, the SixO array $\mathcal{Y}$ is ill-conditioned because of the high collinearity between factors in all modes. Figure 4 shows that contrary to the previous experiment, the considered methods seem to be less effective even for high SNR, especially the ELSALS 


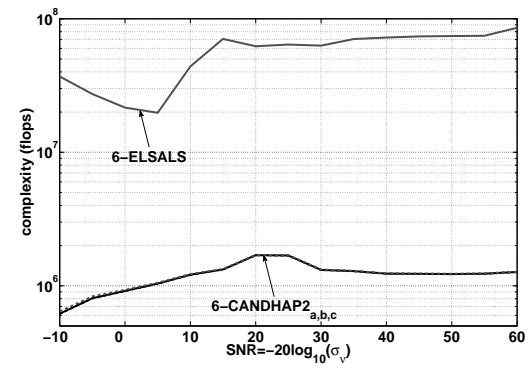

Figure 5: Numerical complexity for the CAND of a SixO Hermitian positive semi-definite array with ill-conditioned loading matrices.

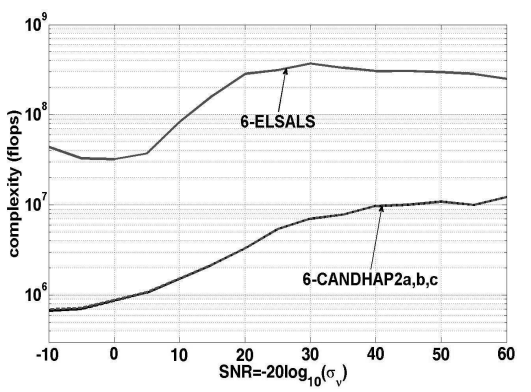

Figure 6: Numerical complexity for the CAND of a SixO Hermitian positive semi-definite array with very ill-conditioned loading matrices.

method. However, even in such a difficult situation, the CANDHAP2 method is more reliable than ELSALS. Note that the initialization procedure used in these experiments is the same as the one adopted in the previous experiment.

\subsubsection{Complexity study}

The computational complexity of the proposed CANDHAP2 and ELSALS algorithms is also evaluated as a function of SNR thanks to the cost expressions given in section 5 using the numbers of iterations obtained from the computer results presented in section 6.2.1. Therefore, the same scenario used in the previous section was adopted here. For $\Delta \phi=25^{\circ}$, figure 5 shows that the proposed CANDHAP2 method is less expensive than the ELSALS method. This can be explained, on the one hand, by the fact that contrary to ELSALS, whatever the order of the original array $\mathcal{Y}$ to be canonically decomposed, CANDHAP2 reduces the iterative decomposition of $\mathcal{Y}$ to that of a third order array $\tilde{\mathcal{T}}$ (27). On the other hand, it derives from the fast convergence of CANDHAP2, which requires fewer iterations, especially for high SNR values.

In a more difficult situation where $\Delta \phi=15^{\circ}$, it is not surprising that all considered methods show a higher numerical complexity compared to the first experiment as displayed in figure 6. Indeed, a higher number of iterations is required in the presence of very close factors. 


\section{Application to blind underdetermined mixture identification}

This section is devoted to evaluate the performance of the proposed ELSALS and CANDHAP2 methods in the context of BUMI. Note that a mixture is called underdetermined when the number of sources exceeds the number of sensors. Let $\boldsymbol{x}$ be an $N$-dimensional random vector modeling $K$ vector observations acquired through $N$ sensors such that:

$$
\boldsymbol{x}=\boldsymbol{A s}+\boldsymbol{\nu}
$$

where $s$ is a $P$-dimensional random vector of sources supposed to be statistically independent, $\boldsymbol{A}$ is the mixing matrix of size $(N \times P)$ and $\boldsymbol{\nu}$ is an $N$-dimensional vector of Gaussian noise independent from $s$. Then, the BUMI problem consists in estimating the potentially underdetermined mixing matrix, $\boldsymbol{A}$, using only the $K$ available realizations of $\boldsymbol{x}$. As mentioned previously, HO cumulants are often used to solve this problem $[13,35,36]$. In fact, cumulants are of great interest in signal processing due to their attractive properties that make them easy to handle. In this section we are interested in $\mathrm{SixO}$ circular cumulant array $\mathcal{C}_{6, \boldsymbol{x}}$ of the observation vector $\boldsymbol{x}$, whose elements $\mathcal{C}_{i, j, k, \boldsymbol{x}}^{\ell, m, n}$ are given in [13, appendix D]. Under the multilinearity property of cumulants and the previous assumptions on $\boldsymbol{x}, \boldsymbol{s}$ and $\boldsymbol{\nu}$, the SixO cumulant array of $\boldsymbol{x}$ is given by:

$$
\mathcal{C}_{6, \boldsymbol{x}}=\sum_{p=1}^{P} \mathcal{C}_{6, s_{p}} \boldsymbol{a}_{p} \circ \boldsymbol{a}_{p} \circ \boldsymbol{a}_{p} \circ \boldsymbol{a}_{p}^{*} \circ \boldsymbol{a}_{p}^{*} \circ \boldsymbol{a}_{p}^{*}
$$

where $\mathcal{C}_{6, s_{p}}$ and $\boldsymbol{a}_{p}$ stand for the real-valued SixO marginal cumulant of the $p$-th source and the $p$-th column vector of the mixing matrix $\boldsymbol{A}$, respectively. Note that according to definition $5, \mathcal{C}_{6, \boldsymbol{x}}$ is Hermitian. It appears from (40) that the $P$ columns, $\boldsymbol{a}_{p}$, of $\boldsymbol{A}$ can be identified by computing the CAND of $\mathcal{C}_{6, \boldsymbol{x}}$. In practice, the exact cumulant array $\mathcal{C}_{6, \boldsymbol{x}}$ is not available and need to be estimated from the $K$ realizations of $\boldsymbol{x}$ using sample statistics [13, section 3.3]. In our experiment, $K$ realizations of $P=4$ Quadrature Phase Shift Keying (QPSK) sources linearly modulated with a pulse shape filter corresponding to a $1 / 2$-Nyquist filter with a roll-off of 0.3 are generated. The sources are supposed to impinge on a UCA of $N=3$ sensors with source DOAs equal to $\gamma_{1}=\gamma_{2}=\gamma_{3}=\gamma_{4}=0$ and $\phi_{1}=10^{\circ}, \phi_{2}=40^{\circ}, \phi_{3}=70^{\circ}$ and $\phi_{4}=100^{\circ}$, which allow us, thanks to equation (34), to mathematically compute the components of the mixing matrix $\boldsymbol{A}$ and to derive $K$ 


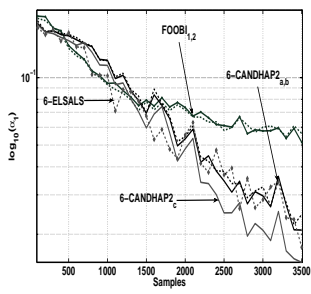

(a) source 1

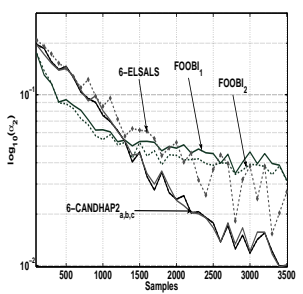

(b) source 2

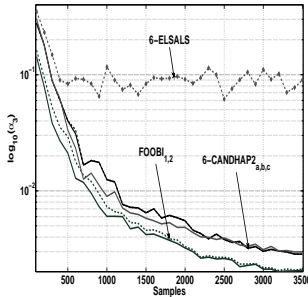

(c) source 3

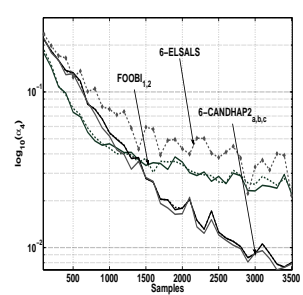

(d) source 4

Figure 7: Criterion $D$ at the output of 6-CANDHAP2 and 6-ELSALS for a UCA of three $N=3$ sensors and four $P=4$ well-angularly equispaced QPSK sources with the same $\mathrm{SNR}=15 \mathrm{~dB}$.

noisy realizations of $\boldsymbol{x}$ using an additive Gaussian noise. All sources have the same SNR equal to $15 \mathrm{~dB}$. Note that, since $\mathcal{C}_{6, s_{1}}=\mathcal{C}_{6, s_{2}}=\mathcal{C}_{6, s_{3}}=\mathcal{C}_{6, s_{4}}=4, \mathcal{C}_{6, \boldsymbol{x}}$ (40) is Hermitian positive semi-definite. Thus, the CANDHAP2 algorithm is appropriate to solve the considered BUMI problem. Regarding the performance criterion, it is the pseudo-distance between mixture $\boldsymbol{A}$ and its estimate $\hat{\boldsymbol{A}}$, defined by $D(\boldsymbol{A}, \hat{\boldsymbol{A}})=\left(\alpha_{1}, \alpha_{2}, \cdots, \alpha_{P}\right)$ where $\alpha_{p}=\min _{1 \leqslant i \leqslant P} d\left(\boldsymbol{a}_{p}, \widehat{\boldsymbol{a}}_{i}\right)$ with $d$ the pseudo-distance between vectors given by [35]:

$$
d(\boldsymbol{u}, \boldsymbol{v})=1-\left\|\boldsymbol{u}^{\mathrm{H}} \boldsymbol{v}\right\|^{2} /\|\boldsymbol{u}\|^{2}\|\boldsymbol{v}\|^{2}
$$

Figure 7 shows the variations of criterion $D$ at the output of $\mathrm{FOOBI}_{1}[36], \mathrm{FOOBI}_{2}$ [36], 6-CANDHAP2 and 6-ELSALS as a function of the data length $K$. We see in the latter figure the higher identification performance of 6-CANDHAP2 compared to 6-ELSALS especially for the second and the fourth sources. Indeed, we note that while 6-CANDHAP2 requires around 1500 data samples to attain a resolution of $10^{-2}$ in identifying the fourth source (see figure 7(d)), 6-ELSALS exhibits a resolution value close to $10^{-1}$ even for a high number of data samples. Regarding the identification of the first and the third sources (see figures $7(\mathrm{a})$ and $7(\mathrm{c})$ ), we note a quasi-similar behavior of 6 CANDHAP $2_{a, b}$ and 6-ELSALS with a slight superiority of 6-CANDHAP $2_{c}$. Eventually, note the good behavior of 6-CANDHAP2 with respect to both FOOBI techniques. 


\section{Conclusion}

Both algorithms proposed in this paper belong to the same family of canonical decomposition methods, namely the family of Alternating Least Squares (ALS) techniques. The main difference between them is the characteristics of the array to be decomposed that they use and the way of using them. Particularly, our generalization of the ELSALS method slightly uses symmetries of the array by computing the same relaxation factor for all factor matrices while the CANDHAP algorithm uses strongly symmetries and positive semi-definiteness of the array especially by reformulating the original CAND problem as the CAND of a third array with a unitary factor matrix. By comparing both approaches, we show through computer results in bottleneck-like situations that the CANDHAP technique outperforms the ELSALS approach in terms of performance and numerical complexity. This confirms that i) when some properties on the array are available, they should be used in order to improve the results, and ii) the way of using them is crucial.

\section{Appendix}

\subsection{Proof of equation (15)}

For all $\theta \in \mathbb{R}$ and for $n \in \mathbb{Z}$, Moivre formula is given by:

$$
f(\theta)=\cos (n \theta)+j \sin (n \theta)=(\cos (\theta)+j \sin (\theta))^{n}
$$

where $j^{2}=-1$. On the other hand, according to Newton binomial formula we have:

$$
(\cos (\theta)+j \sin (\theta))^{n}=\sum_{i=0}^{n}(j)^{i}\left(\begin{array}{c}
n \\
i
\end{array}\right) \cos (\theta)^{n-i} \sin (\theta)^{i}
$$

From equation (42) we can write:

$$
\cos (n \theta)=\Re(f(\theta))=\Re\left(\sum_{i=1}^{n}(j)^{i}\left(\begin{array}{l}
n \\
i
\end{array}\right) \cos (\theta)^{n-i} \sin (\theta)^{i}\right)
$$

Let us now perform a change of variable such that $i=2 \ell$, then equation (44) can be written as:

$$
\cos (n \theta)=\Re\left(\sum_{\ell=0}^{\operatorname{Int}(\mathrm{n} / 2)}\left(j^{2}\right)^{\ell}\left(\begin{array}{c}
n \\
2 \ell
\end{array}\right) \cos (\theta)^{n-2 \ell} \sin (\theta)^{2 \ell}\right)=\sum_{\ell=0}^{\operatorname{Int}(\mathrm{n} / 2)}(-1)^{\ell}\left(\begin{array}{c}
n \\
2 \ell
\end{array}\right) \cos (\theta)^{n-2 \ell} \sin (\theta)^{2 \ell}
$$


Similarly:

$$
\sin (n \theta)=\Im(f(\theta))=\Im\left(\sum_{i=1}^{n}(j)^{i}\left(\begin{array}{l}
n \\
i
\end{array}\right) \cos (\theta)^{n-i} \sin (\theta)^{i}\right)
$$

Let us now perform a change of variable such that $i=2 \ell+1$, then equation (46) can be written as:

$$
\begin{aligned}
\sin (n \theta) & =\Im\left(\sum_{\ell=0}^{\operatorname{Int}((\mathrm{n}-1) / 2)}(j)^{(2 \ell+1)}\left(\begin{array}{c}
n \\
2 \ell+1
\end{array}\right) \cos (\theta)^{n-1-2 \ell} \sin (\theta)^{2 \ell+1}\right) \\
& =\sum_{\ell=0}^{\operatorname{Int}((\mathrm{n}-1) / 2)}(-1)^{\ell}\left(\begin{array}{c}
n \\
2 \ell+1
\end{array}\right) \cos (\theta)^{n-1-2 \ell} \sin (\theta)^{2 \ell+1}
\end{aligned}
$$

where $\Re(r)$ and $\Im(r)$ denote the real and the imaginary parts of the complex variable $r$, respectively.

\subsection{Expressions of coefficients $d_{n}$ and $z_{k}$ in equations (11) and (20)}

Updating the magnitude $r$ and the phase $\theta$ of the relaxation factor $\rho=r e^{j \theta}$ is achieved by rooting the two polynomials expressed in equations (11) and (20), respectively. The twelves coefficients $d_{n}$ of equation (11) and the thirteen coeficients $z_{k}$ of equation (20) are given by:

$$
\begin{aligned}
& \left.d_{0}=2\left(F_{6,7}^{R} \cos (\theta)+F_{6,7}^{I}\right) \sin (\theta)\right), \quad d_{1}=F_{6,6}^{R}+2\left(F_{5,7}^{R} \cos (2 \theta)+\beta_{5,7} \sin (2 \theta)\right) \\
& d_{2}=2\left(F_{5,6}^{R} \cos (\theta)+F_{5,6}^{R} \sin (\theta)+F_{4,7}^{R} \cos (3 \theta)+F_{4,7}^{I} \sin (3 \theta)\right) \\
& d_{3}=F_{5,5}^{R}+2\left(F_{4,6}^{R} \cos (2 \theta)+F_{4,6}^{I} \sin (2 \theta)+F_{3,7}^{R} \cos (4 \theta)+F_{3,7}^{I} \cos (4 \theta)\right) \\
& \\
& d_{4}=2\left(F_{2,7}^{R} \cos (5 \theta)+F_{2,7}^{I} \sin (5 \theta)+F_{3,6}^{R} \cos (3 \theta)+F_{3,6}^{I} \sin (3 \theta)+F_{4,5}^{R} \cos (\theta)+F_{4,5}^{I} \sin (2 \theta)\right) \\
& d_{5}=2\left(F_{1,7}^{R} \cos (6 \theta)+F_{1,7}^{I} \sin (6 \theta)+F_{2,6}^{R} \cos (4 \theta)+F_{2,6}^{I} \sin (4 \theta)+F_{3,5}^{R} \cos (2 \theta)+F_{3,5}^{I} \sin (2 \theta)\right) \\
& d_{6}=2\left(F_{1,6}^{R} \cos (5 \theta)+\beta_{1,6} \sin (5 \theta)+F_{2,5}^{R} \cos (3 \theta)+F_{2,5}^{R} \sin (3 \theta)+F_{3,4}^{R} \cos (\theta)+F_{3,4}^{I} \sin (\theta)\right) \\
& d_{7}=F_{3,3}^{R}+2\left(F_{1,5}^{R} \cos (4 \theta)+F_{1,5}^{I} \sin (4 \theta)+F_{2,4}^{R} \cos (2 \theta)+F_{2,4}^{R} \sin (2 \theta)\right) \\
& d_{8}=2\left(F_{1,4}^{R} \cos (3 \theta)+F_{1,4}^{I} \sin (3 \theta)+F_{2,3}^{R} \cos (\theta)+F_{2,3}^{I} \sin (\theta)\right) \\
& d_{9}=F_{2,2}^{R}+2\left(F_{1,3}^{R} \cos (2 \theta)+F_{1,3}^{I} \sin (2 \theta)\right), \quad d_{10}=2\left(F_{1,2}^{R} \cos (\theta)+F_{1,2}^{I} \sin (\theta)\right), \quad d_{11}=F_{1,1}^{R}
\end{aligned}
$$


and:

$$
\begin{aligned}
z_{0} & =4 p_{10}+5 p_{11}+6 p_{12}+p_{7}+2 p_{8}+3 p_{9}, \quad z_{1}=-2 p_{1}-8 p_{2}-18 p_{3}-32 p_{4}-50 p_{5}-72 p_{6} \\
z_{2} & =-104 p_{10}-220 p_{11}+396 p_{12}+4 p_{7}-4 p_{8}-36 p_{9} \\
z_{3} & =-10 p_{1}-24 p_{2}+6 p_{3}+160 p_{4}+550 P_{5}+1320 p_{6} \\
z_{4} & =60 p_{10}+825 p_{11}+2970 p_{12}+5 p_{7}-34 p_{8}-81 p_{9} \\
z_{5} & =-20 p_{1}-16 p_{2}+108 p_{3}+192 p_{4}-660 p_{5}-4752 p_{6} \\
z_{6} & =336 p_{10}-5544 p_{12}-56 p_{8}, \quad z_{7}=-20 p_{1}+16 p_{2}-108 p_{3}+192 p_{4}-660 p_{5}+4752 p_{6} \\
z_{8} & =60 p_{10}-825 p_{11}+2970 p_{12}-5 p_{7}-34 p_{8}+81 p_{9} \\
z_{9} & =-10 p_{1}+24 p_{2}+6 p_{3}-160 p_{4}+550 p_{5}-1320 p_{6} \\
z_{10} & =-104 p_{10}+220 p_{11}-396 p_{12}-4 p_{7}-4 p_{8}+36 p_{9}, \quad z_{11}=-2 p_{1}+8 p_{2}-18 p_{3}+32 p_{4}-50 p_{5}+72 p_{6} \\
z_{12} & =4 p_{10}-5 p_{11}-p_{7}+2 p_{8}-3 p_{9}+6 p_{12}
\end{aligned}
$$

where the twelve coefficients $p_{n}$ are given by:

$$
\begin{aligned}
& p_{0}=F_{7,7}^{R}+F_{6,6}^{R} r^{2}+F_{5,5}^{R} r^{4}+F_{4,4}^{R} r^{6}+F_{3,3}^{R} r^{8}+F_{2,2}^{R} r^{10}+F_{1,1}^{R} r^{12} \\
& p_{1}=2\left(F_{1,2}^{R} r^{11}+F_{2,3}^{R} r^{9}+F_{3,4}^{R} r^{7}+F_{4,5}^{R} r^{5}+F_{5,6}^{R} r^{3}+F_{6,7}^{R} r\right) \\
& p_{2}=2\left(F_{1,3}^{R} r^{10}+F_{2,4}^{R} r^{8}+F_{3,5}^{R} r^{6}+F_{4,6}^{R} r^{4}+F_{5,7}^{R} r^{2}\right), p_{3}=2\left(F_{1,4}^{R} r^{9}+F_{2,5}^{R} r^{7}+F_{3,6}^{R} r^{5}+F_{4,7}^{R} r^{3}\right) \\
& p_{4}=2\left(F_{1,5}^{R} r^{8}+F_{2,6}^{R} r^{6}+F_{3,7}^{R} r^{4}\right), p_{5}=2\left(F_{1,6}^{R} r^{7}+F_{2,7}^{R} r^{5}\right), p_{6}=2 F_{1,7}^{R} r^{6} \\
& p_{7}=2\left(F_{1,2}^{I} r^{11}+F_{2,3}^{I} r^{9}+F_{3,4}^{I} r^{7}+F_{4,5}^{I} r^{5}+F_{5,6}^{I} r^{3}+F_{6,7}^{I} r\right) \\
& p_{8}=2\left(F_{1,3}^{I} r^{10}+F_{2,4}^{I} r^{8}+F_{3,5}^{I} r^{6}+F_{4,6}^{I} r^{4}+F_{5,7}^{I} r^{2}\right), p_{10}=2\left(F_{1,5}^{I} r^{8}+F_{2,6}^{I} r^{6}+F_{3,7}^{I} r^{4}\right) \\
& p_{9}=2\left(F_{1,4}^{I} r^{9}+F_{2,5}^{I} r^{7}+F_{3,6}^{I} r^{5}+F_{4,7}^{I} r^{3}\right), p_{11}=2\left(F_{1,6}^{I} r^{7}+F_{2,7}^{I} r^{5}\right), p_{12}=2 F_{1,7}^{I} r^{6}
\end{aligned}
$$

\section{Acknowledgement}

This work has been partly supported by: (1) the French ANR contract 10-BLANMULTimODEL, (2) Research Council K.U.Leuven: GOA-MaNet, CoE EF/05/006 Optimization in Engineering (OPTEC), CIF1 and STRT1/08/023, (3) F.W.O.: (a) project G.0427.10N, (b) Research Communities ICCoS, ANMMM and MLDM, (4) the Belgian Federal Science Policy Office: IUAP P6/04 (DYSCO, "Dynamical systems, control and optimization", 2007-2011) and (5) EU: ERNSI. 
[1] L. R. TUCKER, The extension of factor analysis to three-dimensional matrices, Psychometrika 31 (1966) 279-311.

[2] L. DE LATHAUWER, B. DE MOOR, J. VANDEWALLE, A multilinear singular value decomposition, SIAM Journal on Matrix Analysis and its Applications 21 (4) (2000) 1253-1278.

[3] K. YADANI, K. KONDO, M. IWASAKI, A singular value decomposition algorithm based on solving hyperplane constrained nonlinear systems, Applied Mathematics and Computation 216 (3) (2010) 779-790.

[4] P. COMOn, B. MOURRAin, Decomposition of quantics in sums of powers of linear forms, Signal Processing, Elsevier 53 (2) (1996) 93-107, special issue on High-Order Statistics.

[5] F. L. HITCHCOCK, The expression of a tensor or a polydiac as a sum of products, J. Math. Phys. 6 (1) (1927) 164-189.

[6] F. L. HITCHCOCK, Multiple invariants and generalized rank of a $p$-way matrix or tensor, J. Math. Phys. 7 (1) (1927) 39-79.

[7] J. CARROLL, J. CHANG, Analysis of individual differences in multidimensional scaling via an n-way generalization of Eckart-Young decomposition, Psychometrika (9) (1970) 267-283.

[8] A. SMilde, R. BRO, P. GELADI, Multi-way Analysis, Wiley, 2004.

[9] P. KroonenberG, Applied multiway data analysis, Probabil. Statist., New York, Wiely, 2008.

[10] C. S. HERMANN, J. PARNAS, M. MORUP, L. K. HANSEN, S. M. ARNFRED, Parallel factor analysis as an exploratory tool for wavelet transformed event related EEG, NeuroImage 29 (3) (2006) 938-947.

[11] P. COMON, M. RAJIH, Blind identification of underdetermined mixtures based on the characteristic function, Signal Processing, Elsevier 86 (9) (2006) 2271-2281.

[12] B. Chen, A. P. PETROPUlU, Frequency domain blind MIMO system identification based on second- and higher-order statistics, IEEE Transactions On Signal Processing 49 (8) (2001) 16771688 .

[13] L. AlberA, A. FERreOl, P. COMOn, P. CHEVAlieR, Blind Identification of Overcomplete Mixtures of sources (BIOME), Linear Algebra and its Applications 391C (2004) 3-30.

[14] C. E. R. FERnANDES, G. FAVIER, J. C. M. MOTA, Blind channel identification algorithms based on the PARAFAC decomposition of cumulant tensors: the single and multiuser cases, Signal Processing, Elsevier 88 (6) (2008) 1382-1401.

[15] L. DE LATHAUWER, A link between the canonical decomposition in multilinear algebra and simultaneous matrix diagonalization, SIAM Journal on Matrix Analysis and its Applications 28 (3) (2006) 642-666.

[16] J. B. KRUSKAL, Three-way arrays : Rank and uniqueness of trilinear decompositions, Linear Algebra and its Applications 18 (1977) 95-138.

[17] E. SANCHEZ, B. R. KOWALSKI, Generalized rank annihilation factor-analysis, Analytical Chemistry 58 (2) (1986) 496-499.

[18] L. DE LATHAUWER, B. DE MOOR, J. VANDEWALLE, Computation of the canonical decomposition by means of a simultaneous generalized schur decomposition, SIAM Journal on Matrix 
Analysis and Applications 26 (2004) 295-327.

[19] E. ACAR, D. M. DUNLAVY, T. G. KOLDA, A scalable optimization approach for fitting canonical tensor decompositions, Journal of ChemometricsIn press.

[20] R. BRO, C. A. ANDERSSON, Improving the speed of multiway algorithms. Part II: Compression, Chemometrics and Intelligent Laboratory Systems 42 (1-2) (1998) 105-113.

[21] G. TOMASI, R. BRO, PARAFAC and missing values, Chemometrics and Intelligent Laboratory Systems 75 (2004) 163-180.

[22] G. TOMASI, R. BRO, A comparison of algorithms for fitting the parafac model, Computational Statistics and Data Analysis 50 (7) (2006) 1700-1734.

[23] P. COMON, X. LUCIANI, A. L. F. DE ALMEIDA, Tensor decomposition, alternating least squares and other tales, Journal of Chemometrices.

[24] R. A. HARSHMAN, M. E. LUNDY, PARAFAC: Parallel factor analysis, Computational Statistics \& Data Analysis 18 (1) (1994) $39-72$.

[25] J. H. JIANG, H. L. WU, Y. LI, R. Q. YU, Three-way data resolution by alternating slice-wise diagonalization (ASD) method, Journal of Chemometrics 1 (14) (2000) 15-36.

[26] Z. P. CHEN, H. L. WU, H. J. JIANG, Y. LI, R. Q. YU, A novel trilinear decomposition algorithm for second-order linear calibration, Chemometrics and Intelligent Laboratory Systems 52 (1) (2000) $75-86$.

[27] M. RAJIH, P. COMON, R. A. HARSHMAN, Enhanced line search : A novel method to accelerate PARAFAC, Journal on Matrix Analysis and its Applications 30 (3) (2008) 1148-1171.

[28] R. BRO, Multi-way analysis in the food industry: Models, algorithms, and applications, Ph.D. thesis, University of Amsterdam (1998).

[29] R. A. HARSHMAN, Foundation of PARAFAC procedure: Models and conditions for an 'explanatory' multi-mode factor analysis, UCLA working papers in Phonetics (16) (1970) 1-84.

[30] D. NION, L. DE LATHAUWER, An enhanced line search scheme for complex-valued tensor decompositions. application in DS-CDMA, Signal Processing (88) (2008) 749-755.

[31] M. DE VOS, D. NION, S. VAN HUFFEL, L. DE LATHAUWER, A combination of parallel factor and independent component analysis, Internal Report 09-04, ESAT-SISTA, K.U.Leuven, Lirias number: 253441.

[32] G. H. GOLUB, C. F. VAN LOAN, Matrix computations, second edition, The Johns Hopkins University Press, Baltimore, MD, 1989.

[33] A. KARFoul, L. AlBerA, L. DE LATHAuWER, Canonical decomposition of even higher order cumulant arrays for blind underdetermined mixture identification, in: SAM 2008, The fifth IEEE Sensor Array and Multichannel Signal Processing workshop, Darmstatdt, Germany, 2008, pp. 501505.

[34] L. AlberA, A. KARFoul, L. DE LATHAUWER, Décomposition canonique de tableaux hermitiens semi-définis positifs d'ordre pair par rotation procustéenne : application à l'ICA, in: 22ième Colloque GRETSI sur le Traitement du Signal et des Images (GRETSI 2009), Dijon, France, 2009.

[35] A. FERREOL, L. ALBERA, P. CHEVALIER, Fourth order blind identification of underdetermined 
mixtures of sources (FOBIUM), IEEE Transactions On Signal Processing 53 (2005) 1254-1271.

[36] L. DE LATHAUWER, J. CASTAING, J.-F. CARDOSO, Fourth-order cumulant-based blind identification of underdetermined mixtures, IEEE Transactions on Signal Processing 55 (6) (2007) 29652973.

[37] L. DE LATHAUWER, B. DE MOOR, J. VANDEWALLE, On the best rank-1 and rank$(\mathrm{R} 1, \mathrm{R} 2, \ldots, \mathrm{RN})$ approximation of high-order tensors, Journal Matrix Analysis and its Applications 21 (4) (2000) 1324-1342.

[38] P. COMON, J. M. F. TEN BERGE, L. DE LATHAUWER, J. CASTAING, Generic and typical ranks of multi-way arrays, Linear Algebra and its Applications 430 (11-12) (2009) 2997-3007.

[39] A. STEGEMAN., N. D. SIDIROPOUlOS, On kruskal's uniqueness condition for the CANDECOMP/PARAFAC decomposition, Linear Algebra and its applications 420 (2-3) (2007) 540-552.

[40] R. CATTELL, Parallel proportional profiles and other priciples for determining the choice of factors by rotation, Psychometrika 9 (4) (1944) 267-283.

[41] N. D. SIDIROPOULOS, R. BRO, On the uniqueness of multilinear decomposition of N-way arrays, Journal of Chemometrics 14 (2000) 229-239.

[42] N. D. SIDIROPOULOS, R. BRO, On the uniqueness of the multilinear decomposition of n-way arrays, Journal of chemometrics 14 (2000) 229-239.

[43] N. D. SIDIROPOUlOS, R. BRO, G. B. GIANNAKIS, Parallel factor analysis in sensor array processing, IEEE Transactions On Signal Processing 48 (8) (2000) 2377-2388.

[44] T. JIANG, N. D. SIDIROPOULOS, Kruskal's permutation lemma and the identification of CANDECOMP/PARAFAC and bilinear models with constant modulus constraints, IEEE Transaction on Signal Processing 52 (9) (2004) 2625-2636.

[45] M. MELLA, Singularities of linear systems and the waring problem, Transactions of the American Mathematical Society 358 (12) (2006) 5523-5538.

[46] T. LICKTEIG, Typical tensorial rank, Linear Algebra and its Applications 69 (1985) 95-120.

[47] C. NIKIAS, A. P. PETROPULU, Higher Order Spectra Analysis: A Non-Linear Signal Processing Framework, facsimile Edition, Prentice Hall PTR, 1993.

[48] J. TEN BERGE, Least squares optimization in multivariate analysis, notes Edition, M\&T, DSWO Press, Leiden, The Netherlands, 1993.

[49] J.-F. CARDOSO, A. SOULOUMIAC, Jacobi angles for simultaneous diagonalization, SIAM Journal on Matrix Analysis and Applications 17 (1) (1996) 161-164.

[50] E. M. FADAILI, N. THIRION-MOREAU, E. MOREAU, Non orthogonal joint diagonalization/zerodiagonalization for source separation based on time-frequency distributions, IEEE Transactions On Signal Processing 55 (5) (2007) 1673-1687.

[51] A. YEREDOR, Non-orthogonal joint diagonalization in the least-squares sense with application in blind source separation, IEEE Transactions On Signal Processing 50 (7) (2002) 1545-1553.

[52] R. A. HARSHMAN, M. E. LUNDY, Data preprocessing and the extended PARAFAC model, in: H. G. LAW, C. W. SNYDER, J. A. HATTIE, R. P. MCDONALD (Eds.), Research Methods for Multi-mode Data Analysis, New York: Praeger, 1984, pp. 216-284. 
[53] W. KRIJNEN, T. DIJKSTRA, A. STEGEMAN, On the non-existence of optimal solutions and the occurrence of "degeneracy" in the CANDECOMP/PARAFAC model, Psychometrika 73 (2008) 431-439.

[54] A. STEGEMAN, J. M. F. TEN BERGE, L. DE LATHAUWER, Sufficient conditions for uniqueness in CANDECOMP/PARAFAC and INDSCAL with random component matrices, Psychometrika 71 (2) (2006) 219-229. 